\title{
ON THE PASSAGE FROM LOCAL TO GLOBAL IN NUMBER THEORY
}

\author{
B. MAZUR
}

\section{INTRODUCTION}

Would a reader be able to predict the branch of mathematics that is the subject of this article if its title had not included the phrase "in Number Theory"? The distinction "local" versus "global", with various connotations, has found a home in almost every part of mathematics, local problems being often a stepping-stone to the more difficult global problems.

To illustrate what the earthy geometric terms local and global signify in Number Theory, let us consider any Diophantine equation, say,

$$
X^{n}+Y^{n}+Z^{n}=0 \quad(n>2) .
$$

Determining the rational (or equivalently, integral) solutions of such equations can be difficult! In contrast, to determine its solutions in integers modulo $m$ (at least, for any fixed modulus $m>0$ ) is a finite problem-a comparatively easy problem. Moreover, this easy problem, thanks to the Chinese Remainder Theorem, can be replaced by the problem of finding its solutions modulo the prime powers $p^{\nu}$ dividing $m$. Given an integral solution, i.e., $a^{n}+b^{n}+c^{n}=0$, for $a, b, c \in \mathbb{Z}$, by viewing $(a, b, c)$ as a triple of integers modulo $p^{\nu}$ for any prime number $p$ and any $\nu \geq 0$, we get, of course, a solution modulo $p^{\nu}$.

Ever since (implicitly) the work of Kummer and (explicitly) the work of Hensel, we know that it is useful to note that an integral solution to such a Diophantine equation yields more: for each prime number $p$, and for positive integers $\nu=1,2,3, \ldots$, if we choose to view the integral solution, successively, as a solution modulo $p^{\nu}$, we then get, for every prime number $p$, a system of solutions modulo $p^{\nu}$ for each $\nu=1,2, \ldots$, the system being "coherent" in the sense that reduction of the $(\nu+1)$ st solution modulo $p^{\nu}$ yields the $\nu$ th solution. Of course, such a system is nothing more than a solution in the projective limit $\mathbb{Z}_{p}:=\operatorname{Lim}\left(\mathbb{Z} / p^{\nu} \mathbb{Z}\right)$, the ring of $p$-adic integers $\left(\mathbb{Z}_{p}\right.$ being considered as a topological ring, given the "profinite topology", i.e., the natural topology that it inherits as a projective limit of the finite discrete rings $\mathbb{Z} / p^{\nu} \mathbb{Z}$ ).

For more flexibility, one can work in $\mathbb{Q}_{p}$, the field of fractions of $\mathbb{Z}_{p}$ (most economically obtained from $\mathbb{Z}_{p}$ by adjoining the inverse of the single element $p$ ). The field $\mathbb{Q}_{p}$ contains $\mathbb{Q}$ and can be regarded as the topological (in fact, metrized) field obtained by completing the field $\mathbb{Q}$ with respect to the " $p$-adic" metric $^{1}$. Evidently then, if a polynomial equation with $\mathbb{Q}$-coefficients fails to

Received by the editors October 1, 1992 and, in revised form, February 17, 1993.

1991 Mathematics Subject Classification. Primary 11-02, 14-02, 14-G05, 14G20, 14G25, 14J20, $14 \mathrm{~K} 15$

${ }^{1}$ Two rational numbers are very close in the $p$-adic metric if their difference, expressed as a fraction in lowest terms, has the property that its numerator is divisible by a high power of $p$. The standardly normalized $p$-adic metric ||$_{p}$ is characterized by the fact that it is multiplicative, that $|p|_{p}=1 / p$, and that if $a$ is an integer not congruent to $0 \bmod p$, then $|a|_{p}=1$. 
have a solution over some $p$-adic field $\mathbb{Q}_{p}$, it cannot have a solution over $\mathbb{Q}$.

The formal relationship between the field $\mathbb{Q}$ and the collection of completions $\mathbb{Q}_{p}$ (for $p$ ranging through all prime numbers) is closely analogous to the relationship between the field $K$ of global rational functions on, say, a smooth algebraic curve $S$ over $\mathbb{C}$ in the affine plane, and the collection of fields $K_{S}$ of formal Laurent series with finite order poles, centered at points $s \in S$. The distinction of global $\mathbf{v}$. local is evident here: any global meromorphic function $f \in K$ gives rise to a collection of local data, namely, the collection of Laurent expansions of $f$ at each of the points $s \in S$. Our affine plane curve $S$ is not compact, and therefore the collection of fields $\left\{K_{s}\right\} f_{s \in S}$ does not reflect "the whole local story". To get "the whole story", we usually augment our collection $\left\{K_{s}\right\}$ by including the completions of $K$ corresponding to the "missing" points, the points at infinity. It is only when one takes account of all the completions $\left\{K_{s}\right\}_{s}$ for all points $s$ in the smooth compactification of $S$ that certain global constraints on the collections of local data hold, the most basic being that the number of zeros of a global function (zeros counted with appropriate multiplicity) is equal to the number of its poles; equivalently, if $\left\{f_{s}\right\}_{s}$ is a collection of Laurent series all coming from the same global meromorphic function $f$, then

$$
\sum \operatorname{ord}_{s}\left(f_{s}\right)=0 \text {, }
$$

where $\operatorname{ord}_{s}\left(f_{s}\right)$ is the order of zero (or, if negative, pole) of the Laurent series $f_{s}$ at $s$.

In analogy, it has long been understood (cf. [W]) that there is one "missing field" which should be considered along with the collection of $p$-adic completions of $\mathbb{Q}$; namely, the field $\mathbb{R}$, of real numbers, i.e., the $U r$-completion of $\mathbb{Q}$ (with respect to the standard absolute value metric, which we denote by ||$_{\infty}$, to bring it notationally into line with the $p$-adic metrics). The analogue of (1) is the following (easily proved) formula ${ }^{2}$ : If $r \in \mathbb{Q}$, then

$$
\sum_{p} \log |r|_{p}=0
$$

provided the summation subscript $p$ ranges over all prime numbers and ("the infinite prime") $\infty$.

One weakness in the analogy between the collection $\left\{K_{s}\right\}_{s \in S}$ for a compact Riemann surface $S$ and the collection $\left\{\mathbb{Q}_{p}\right.$, for prime numbers $p$, and $\left.\mathbb{R}\right\}$ is that the fields $K_{s}$ for all points $s$ are very much alike; they are even isomorphic. In contrast, no manner of squinting seems to be able to make $\mathbb{R}$ the least bit mistakable for any of the $p$-adic fields, nor are the $p$-adic fields $\mathbb{Q}_{p}$ isomorphic for distinct $p$. A major theme in the development of Number Theory has been to try to bring $\mathbb{R}$ somewhat more into line with the $p$-adic fields; a major mystery is why $\mathbb{R}$ resists this attempt so strenuously.

Here are two types of questions in Number Theory one might want to pursue by passing from local to global:

Type (A). Questions about rational points. Given a Diophantine equation or a system of Diophantine equations with coefficients in $\mathbb{Q}$, when does knowledge about its rational solutions over the collection of local fields $\mathbb{Q}_{p}$ for all prime

\footnotetext{
${ }^{2}$ Exponentiating both sides of this equation, one gets the somewhat more familiar "product formula".
} 
numbers $p$, and over $\mathbb{R}$, give us some palpable information about its solutions over $\mathbb{Q}$ ?

Here is one of the simplest examples of such a question:

For which classes of systems of Diophantine equations with coefficients in $\mathbb{Q}$ is it the case that the existence of solutions over $\mathbb{Q}_{p}$ for all prime numbers $p$ and over $\mathbb{R}$ guarantees the existence of a solution over $\mathbb{Q}$ ?

The question of existence of solutions of systems of Diophantine equations over $\mathbb{R}$ or over $\mathbb{Q}_{p}$ is "certifiably easy"; at least it is a decidable question in the sense of mathematical logic. In fact, all first-order theories over $\mathbb{R}$ (or over $\mathbb{Q}_{p}$ ) are decidable, this being a result due to Tarski (and Ax-Kochen), a concise treatment of which can be found in [Coh]. Whether or not the question of existence of solutions of systems of Diophantine equations over $\mathbb{Q}$ is a decidable question is still up in the air ${ }^{3}$, but, as the example which we trotted out at the beginning of this article was meant to convince, decidable or not, even specific polynomials with coefficients in $\mathbb{Q}$ can require quite elaborate theory before we can say that all their $\mathbb{Q}$-rational points are known ${ }^{4}$.

Type (B). Passing from knowledge about local "structures" to knowledge about global "structures". There is a wealth of literature on various aspects of questions of type $(\mathrm{A})^{5}$. One cannot, really, effectively "separate" questions of type (A) from questions of type (B), but the central aim of this article is to discuss an exciting development (finiteness of certain Tate-Shafarevich groups ${ }^{6}$ ), which is conveniently expressed in the language of $(B)$.

In 1986 at the MSRI, Larry Washington told Karl Rubin of some new ideas of Thaine, suspecting that they might be helpful in Rubin's work. These ideas, as formulated by Thaine ${ }^{7}$, had been brought to bear on questions concerning the ideal class groups of cyclotomic fields [Th]. Rubin refashioned Thaine's ideas to serve as a tool in the study of the arithmetic of complex multiplication elliptic curves. By their means, Rubin was able, among other things, to prove that some Tate-Shafarevich groups of elliptic curves over number fields were finite. This provided us with the first examples (in the number field case ${ }^{8}$ ) where the finiteness of some Tate-Shafarevich groups was actually proved, despite the fact that (all) Tate-Shafarevich groups had been conjectured, three decades ago, to be finite.

Soon thereafter, working independently, V. A. Kolyvagin devised his approach to these finiteness questions (Kolyvagin's method of "Euler Systems" resembles the method of Thaine-Rubin but is more flexible and is applicable to a much broader range of problems).

\footnotetext{
${ }^{3}$ Matijasevic has established the algorithmic undecidability of the question of whether any given Diophantine equation has an integral solution [Mat].

${ }^{4}$ Anyone who doubts this should try to read published accounts of the proof of "Fermat's Last Theorem" for exponent $n=37$.

${ }^{5}$ For example, for a recent article which contains, among other things, an extensive review and bibliography concerning current activities concerning the Hasse principle and "weak approximation" (of local solutions by global ones) in some classes of varieties, see [C-T-S-D].

${ }^{6}$ We will be explaining this.

${ }^{7}$ Thaine says that at least partial inspiration for these ideas came from a close reading of a paper of Kummer.

${ }^{8}$ There had already been some function field examples [Mi1-3].
} 
Kolyvagin's ideas (and those of Rubin and Thaine) represent something of a revolution ${ }^{9}$.

My plan in Part I is to try to explain to a general audience some of the importance of Finiteness of the Tate-Shafarevich Group. Anything discussed in Part I that smacks of being too technical is tucked into footnotes or into Part III.

Part II is an analytic continuation of Part I in which I try to give a bit of the flavor of how one goes about showing such finiteness. It is written in more technical language than Part I, but still no proofs are given here. I hope that it may be useful to some readers as an accompaniment to some of the published articles on the subject or as a companion to some of the other surveys; see, for example, the recent [ $\mathrm{Ne} 2]$.

Part III provides proofs of some things that seemed natural to bring up in an expository treatment of this sort, results that are, very likely, well known to the experts but for which there is no reference in the literature.

\section{Contents}

\section{Part I}

1. Local-to-global principles (an example of a question of type (A): zeroes of quadratic forms)

2. Local-to-global principles (an example of a question of type (B): isomorphisms of projective varieties)

3. First examples: projective space, quadrics, and curves of genus zero

4. Curves of genus $\geq 1$. The "local-to-global principle up to finite obstruction"

5. General projective varieties

6. The Tate-Shafarevich group

7. Implications of the Tate-Shafarevich Conjecture in the direction of the general local-to-global principle (up to finite obstruction)

8. Examples of finiteness of the Tate-Shafarevich group

9. Returning to Selmer's curve

\section{Part II}

10. The Tate-Shafarevich group and the Selmer group

11. Local control of the Selmer group

\footnotetext{
${ }^{9}$ The impressive number of otherwise inaccessible problems that these methods successfully treat is one measure of the revolutionary aspect of this work; the bibliography at the end of these notes gives a small sample of the activity involved here. For example, thanks to Kolyvagin and Rubin we now have: (1) a significantly shorter proof of the "Main Conjecture over $\mathbb{Q}$ " (originally proved by Andrew Wiles and me); (2) the one- and two-variable "Main Conjecture" over quadratic imaginary fields; and (3) (most of) the Birch Swinnerton-Dyer Conjecture for modular elliptic curves whose $L$ function does not have a multiple zero at $s=1$, and in particular, that (for these elliptic curves) the Tate-Shafarevich group is finite (see Theorem 3 ).

But even more telling is that, after all this, we now think of the subject in quite a different way, and a range of new arithmetic problems seems nowadays to be within reach. For example, there is Nekovár's recent study of Selmer groups attached to higher (even) weight to gain information about algebraic cycles on Kuga-Sato varieties [Ne1]; there is Flach's finiteness theorem for certain Selmer groups attached to automorphic forms arising as the symmetric square of classical modular forms which yields information about the deformation space of the Galois representations attached to classical forms [F]; there is Darmon's attack on the "refined conjectures" of Birch SwinnertonDyer type [D1, D1] (see also [BD]); there is Kato's recent exciting announcement concerning the construction of Kolyvagin-type ("Euler") systems of classes in algebraic $K$-groups.
} 
12. Class field theory, duality, and Kolyvagin test classes

13. Rational points in extension fields yielding "test classes"

14. Heegner points

Part III

15. The cohomology of locally algebraic group schemes

16. Discrete locally algebraic group schemes

17. Finiteness theorems

18. The mapping of the Tate-Shafarevich group of $A / K$ to $\mathscr{S}(A / K)$

19. The local-to-global principle for quadrics

Bibliography

\section{PART I}

1. Local-to-global principles (an example of a question of type $(A)^{10}$ : zeros of quadratic forms). The Theorem of Hasse-Minkowski [H] guarantees that

a quadratic form with coefficients in $\mathbb{Q}$ admits a nontrivial zero over $\mathbb{Q}$ if and only if it does so over $\mathbb{Q}_{p}$ for all prime numbers $p$ and over $\mathbb{R}$.

To get a closer view of the sort of information that this classical result gives us, let us think about it in the following special case: Consider a diagonal quadratic form in three variables

$$
F(X, Y, Z)=a \cdot X^{2}+b \cdot Y^{2}-c \cdot Z^{2}
$$

with $a, b, c$ positive integers.

The fact that the coefficients in (1) are not all of the same sign guarantees that our form has a nontrivial zero over $\mathbb{R}$. The Theorem of Chevalley (which says that a polynomial in more variables than its degree, with integer coefficients $\bmod p$ and no constant term has a nontrivial zero $\bmod p)$ gives us that $(1)$ has a nontrivial zero modulo $p$ for any prime number $p$. Moreover, for odd prime numbers $p$ not dividing $a \cdot b \cdot c$, Hensel's Lemma then tells us that the nontrivial zero $\bmod p$ given to us by Chevalley's theorem lifts to a nontrivial $p$-adic zero. Thus, in this case, the Hasse-Minkowski Theorem assures us that, if

$$
a \cdot X^{2}+b \cdot Y^{2}-c \cdot Z^{2}=0
$$

has a nontrivial $p$-adic solution for the finite set of primes $p$ dividing $2 \cdot a \cdot b \cdot c$, then it has a rational solution.

There is no difficulty in stipulating congruence conditions on $a, b, c$ under which (2) will have $p$-adic solutions for this finite set of prime numbers $p$.

But even in a concrete instance of (2), where the Hasse-Minkowski Theorem guarantees the existence of a rational solution, there remains much yet to ponder about; for the proof of the Hasse-Minkowski Theorem does not produce any specific nontrivial rational solution easily, despite the fact that the problem of finding such solutions is perfectly effective (e.g., in Mordell's book [Mo] it is shown that if (2) has a nontrivial rational solution, it has such a solution with

$$
|X| \leq \sqrt{b \cdot c}, \quad|Y| \leq \sqrt{c \cdot a}, \quad|Z| \leq \sqrt{a \cdot b} .
$$

For other, more general bounds see [Ca1, 6.8, Lemma 8.1]).

\footnotetext{
${ }^{10}$ Type (A) refers to the distinction alluded to in the discussion in the introduction.
} 
As Victor Miller pointed out, it would be good to give explicit bounds for the adelic version of this problem, i.e., given a finite set of prime numbers $p$ and specific $p$-adic points for these $p$, find rational points of prescribed closeness to these $p$-adic points.

Moreover, it would be of interest to specify fast algorithms (e.g., are there algorithms which are of polynomial time in $\max (\log a, \log b, \log c)$ ?) for the determination of these rational points.

For recent results concerning the equidistribution properties of the sets $\mathscr{S}_{g}(n)$ of integral solutions of $g(x, y, z)=n$ (for specified integers $n$ ) where $g(x, y, z)$ is a positive-definite ternary quadratic form (equidistribution of the sets $\mathscr{S}_{g}(n)$, that is, when they are scaled back to the unit ellipsoid $g(x, y, z)=$ 1 in $\mathbb{R}^{3}$ ), see [Du, GF, D-S-P] ${ }^{11}$.

There are also distributional types of estimates coming from the circle method concerning rational zeros of diagonal forms (in four or more variables). For example, Theorem 4 of Siegel's 1941 paper [Si] treats the special case of a nondegenerate quadratic form in four variables with integral coefficients,

$$
F(X, Y, Z, T)=a X^{2}+b Y^{2}+c Z^{2}+d T^{2},
$$

whose determinant is the square of an integer. Explicitly, fix such a quadratic form $F$, and for $S=(X, Y, Z, T)$ a four-tuple of integers, put

$$
\|S\|:=|a| \cdot X^{2}+|b| \cdot Y^{2}+|c| \cdot Z^{2}+|d| \cdot T^{2} .
$$

Theorem 4 of loc. cit. gives a positive constant $\kappa$ such that

$$
\sum_{S} \exp (-\|S\| / \lambda)=\kappa \cdot \lambda \log \lambda+o(\lambda \log \lambda) \quad(\text { as } \lambda \rightarrow \infty),
$$

the summation being extended over all (nontrivial) integral zeros $S$ of $F$, assuming that $F$ has nontrivial integral zeros (equivalently, assuming that $F$ is indefinite and has a nontrivial zero in $\mathbb{Q}_{p}$ for all prime numbers $p$ ).

2. Local-to-global principles (an example of a question of type $(B)^{12}$ : isomorphisms of projective varieties). One says that two quadratic forms (in the same number of variables) over a field $K$ are equivalent if one can pass from one of the quadratic forms to the other (and from the other to the one) by suitable linear substitutions of variables, where the matrix describing the linear change of variables has coefficients in $K$. Questions of equivalence of quadratic forms over local fields are quite manageable; for example, by "Sylvester's Law", quadratic forms over $\mathbb{R}$ are determined by three integers (their rank, nullity, and index).

Here is a variant of the Hasse-Minkowski theorem (cf. [Has, Si]) expressed as the answer to a question of type (B):

Two quadratic forms with coefficients in $\mathbb{Q}$ which are equivalent over $\mathbb{Q}_{p}$ for all prime numbers $p$ and over $\mathbb{R}$ are equivalent over $\mathbb{Q}$.

In this type (B) spirit, let us prepare for a more supple and more general local-to-global question.

${ }^{11}$ The case of three variables is particularly difficult; these equidistribution results are based on delicate upper bounds for the absolute values of the Fourier coefficients of modular forms of half-integral weight obtained by Iwaniec [I].

${ }^{12}$ Type (B) refers to the distinction alluded to in the discussion in the introduction. 
Let $V$ be a projective variety defined over any field $k$. We can think of $V$ as "given by" some finite collection of homogeneous forms in $N$ variables (for some $N$ ) with coefficients drawn from $k$. The set $V(L)$, of $L$-rational points of $V$, for $L$ any field extension of $k$, is the common "locus of zeros" (in projective $N$-space with homogeneous coordinates in $L$ ) of the collection of homogeneous forms defining $V$. Assume that $V$ is smooth ${ }^{13}$.

The notion of isomorphism between two (smooth) varieties $V$ and $W$ over $k$ (the technical term being biregular isomorphism ${ }^{14}$ defined over $k$ ) will be important to us. But here, in contrast to the notion of equivalence of quadratic forms, one allows changes of variables more fluid than the mere linear changes of variables allowed in the definition of equivalence in the theory of quadratic forms. ${ }^{15}$ We admit the possibility that $V$ and $W$ may be given as loci of zeros of systems of homogeneous forms in different dimensional projective spaces and that the systems for $V$ and for $W$ may comprise different numbers of homogeneous forms and of different degrees. ${ }^{16}$

Now let $V$ be a smooth variety over $\mathbb{Q}$. Embedding $\mathbb{Q}$ in its various completions, we may forget that our $V$ is defined over $\mathbb{Q}$ and think of $V$ as defined over $\mathbb{Q}_{p}$, where $p$ runs through all prime numbers, or over $\mathbb{R}$, by simply regarding the defining collection of homogeneous forms of $V$ as having their coefficients in $\mathbb{Q}_{p}$ or in $\mathbb{R}$. To indicate that we have done this, we shall sometimes refer to the "original" variety $V$, defined over $\mathbb{Q}$ as $V / \mathbb{Q}$; and if we wish to think of it as defined over $\mathbb{Q}_{p}$ or over $\mathbb{R}$, we refer to it as $V / \mathbb{Q}_{p}$ or $V / \mathbb{R}$.

Question. To what extent do the "local" varieties

$$
\left\{V / \mathbb{Q}_{p}, \text { for all } p, \text { and } V / \mathbb{R}\right\}
$$

determine the "global" variety $V / \mathbb{Q}$ (up to isomorphism over $\mathbb{Q}$ )?

To have vocabulary for this, let us say that a (smooth projective) variety $V^{\prime} / \mathbb{Q}$ is a companion to $V / \mathbb{Q}$ if their corresponding local varieties are isomorphic, ${ }^{17}$

\footnotetext{
${ }^{13}$ Why not? "Smoothness" is defined, as in multivariable calculus, by the requirement that the appropriate jacobian determinant does not vanish at $\bar{k}$-rational points of $V$.

${ }^{14}$ See Lecture 2 of [Harr] for the notion of "regular morphism" over algebraically closed fields and [Hart] for the foundations for the more general concept of "morphism of schemes".

${ }^{15}$ Nevertheless, in the special case of (smooth) quadrics, i.e., varieties given as the locus of zeros of a single (nondegenerate) quadratic form, the quadratic forms are similar (i.e., equivalent up to multiplication by a nonzero scalar) over $K$ if and only if the varieties are isomorphic over $K$. The point here is that (setting aside the case of quadric surfaces, for the moment), if $V$ is a smooth quadric of dimension 1 or $\geq 3$, then $\operatorname{Pic}(V)=\mathbb{Z}$. Therefore, any automorphism of the variety $V$ must preserve the isomorphism class of $L$, the (unique) generating ample line bundle, and consequently must extend to projective linear automorphisms of the projective spaces coming from the linear spaces of global sections of $L^{\otimes n}$ for any $n$. Since the initial embedding of $V$ in projective space (via the defining quadratic form) is the projective space of global sections of $L^{\otimes 2}$ if $\operatorname{dim} V=1$, and of $L$ if $\operatorname{dim} V \geq 3$, one easily sees that similarity between such quadratic forms is the same as isomorphism between their corresponding varieties. The same conclusion holds for smooth quadric surfaces $V$ with a slightly different argument (since the Picard group of $V$ over $\bar{K}$ is $\mathbb{Z} \oplus \mathbb{Z})$.

${ }^{16}$ When the field $k$ is $\mathbb{C}$, using the faithfulness of the functor which passes from a smooth projective varieties $V$ over $\mathbb{C}$ to its underlying complex analytic manifold $V(\mathbb{C})[\operatorname{Se} 1]$, one sees that to give a biregular morphism from $V$ to $W$ over $\mathbb{C}$ is equivalent to giving a complex-analytic isomorphism from $V(\mathbb{C})$ to $W(\mathbb{C})$.

${ }^{17}$ Companion is a neologism; twisted $\mathbb{Q}$-form is the term sometimes used, but $I$ prefer a new word here, since the term "twisted form" occurs in a number of different contexts. Moreover, we
} 
i.e., if $V^{\prime} / \mathbb{Q}_{p}$ is isomorphic to $V / \mathbb{Q}_{p}$ (as varieties over $\mathbb{Q}_{p}$ ) for all prime numbers $p$, and $V^{\prime} / \mathbb{R}$ is isomorphic to $V / \mathbb{R}$ (as varieties over $\mathbb{R}$ ).

Denote by $\mathscr{S}(V)$ the set of isomorphism classes over $\mathbb{Q}$ of varieties $V^{\prime} / \mathbb{Q}$ which are companions to $V .{ }^{18}$

One can think of the cardinality of $\mathscr{S}(V)$ as roughly analogous to a class number, i.e., a measure of the extent to which local data (in this case, the isomorphism classes of $V / \mathbb{Q}_{p}$ for all $p$, and $V / \mathbb{R}$ ) determine or fail to determine global data (the isomorphism class of $V / \mathbb{Q}$ ). One might say that the local-toglobal principle holds for a class of varieties $\mathscr{V}$ if $\mathscr{S}(V)$ consists of the single isomorphism class $\{V\}$ for each member $V$ of $\mathscr{V}$.

\section{First examples: projective space, quadrics, and curves of genus zero.}

Projective space. The local-to-global principle holds for any variety $V / \mathbb{Q}$ which is isomorphic (over $\mathbb{C}$ ) to projective $N$-space for some $N$. This statement, ${ }^{19}$ which is one of the many manifestations of the local-to-global principle for elements of the Brauer group ${ }^{20}$ of $\mathbb{Q}$, might be viewed as an arithmetic addendum to the large body of literature (e.g., differential geometric and algebraic geometric) establishing various ways in which projective space is rigid. The analogous statements hold more generally with $\mathbb{Q}$ replaced by any number field.

Quadrics. The local-to-global principle holds for any smooth quadric variety $V$ over $\mathbb{Q}$ (and, again, more generally over any number field). I am thankful to Colliot-Thelène for providing me with a sketch of a proof of this (see Part III, $\S 19)$.

Curves of genus 0 . The local-to-global principle holds for any (smooth, projective) curve of genus 0 which is defined over $\mathbb{Q}$ (or over any number field). This follows from either of the two previously cited examples, since:

(i) any smooth curve of genus 0 over an algebraically closed field is isomorphic to $\mathbf{P}^{1}$, and

(ii) any smooth curve of genus zero over any field $k$ is canonically isomorphic to a plane conic curve over $k .^{21}$

4. Curves of genus $\geq 1$. The "local-to-global principle up to finite obstruction". That the local-to-global principle does not always hold for curves of genus 1 was seen early on, the first explicit examples being given by Lind and Reichart in

will want to reserve the term "twist" (below) for abelian varieties which are twists of other abelian varieties.

${ }^{18}$ So as not to get sidetracked in technical things, we leave for Part III a discussion of the cohomological interpretation of $\mathscr{S}(V)$.

${ }^{19}$ Contained in the $1944 \mathrm{Ph}$.D. thesis of Châtelet, for a historical discussion of which see [C-T, $\S 1]$.

${ }^{20}$ For the Brauer group and the relationship between Brauer groups over local fields and global ones, see [Se3].

${ }^{21}$ This isomorphism is given as the projective embedding associated to the anticanonical line bundle on the smooth curve of genus 0 . Serre suggested that $I$ also point out the fact that the category of quaternionic division algebras over any field $k$ of characteristic $\neq 2$ is equivalent to the category of smooth curves of genus 0 over $k$, an equivalence in one direction being given as follows: If $D$ is a quaternionic division algebra over $k$, let $V_{D}$ denote the plane conic over $k$ given by the equations $\operatorname{Tr}(x)=0$ and $\mathrm{N}(x)=0$ in $D$, where $\operatorname{Tr}$ is the trace and $\mathrm{N}$ is the quaternionic norm. Exercise (Serre). Find an explicit equivalence of categories going in the other direction. 
the early forties. In fact, this principle fails either in its variants type (a) or type (B), for the failure of these two variants are rather close in the context of curves of genus 1: If a curve of genus 1 possesses a $\mathbb{Q}_{p}$-rational point for all prime numbers $p$ and a real point, and it does not have a $\mathbb{Q}$-rational point, then it has at least one nonisomorphic companion. Conversely, if it has a nonisomorphic companion, then at least one of its companions has no $\mathbb{Q}$-rational point (yet has $\mathbb{Q}_{p}$-rational points for all $p$ and real points).

That the local-to-global principle fails even for smooth plane cubic curves ${ }^{22}$ was shown by Selmer (1951). Here is his most widely quoted example:

The curve $C: 3 x^{3}+4 y^{3}+5 z^{3}=0$ has nonisomorphic companions. This equation possesses nontrivial solutions over $\mathbb{Q}_{p}$ for all prime numbers $p$ and over $\mathbb{R}$, but it possesses no nontrivial solutions over $\mathbb{Q}$.

When I was preparing these notes, I initially intended to quote this Selmer example and go on. But then it occurred to me that after the recent work which is the subject of this article, we are actually in a position to explore the question of companions to Selmer's curve (and others) with some precision. Working out such an example seems to me to be a good way of demonstrating the power of the recent results of Rubin and Kolyvagin. So, the following is an explicit list of all the companions (worked out with the help of some e-mail communications of Rubin):

Theorem 1. Selmer's curve $C: 3 x^{3}+4 y^{3}+5 z^{3}=0$ has, counting itself, precisely five companions:

$$
\begin{aligned}
3 x^{3}+4 y^{3}+5 z^{3} & =0, \\
12 x^{3}+y^{3}+5 z^{3} & =0 \\
15 x^{3}+4 y^{3}+z^{3} & =0 \\
3 x^{3}+20 y^{3}+z^{3} & =0 \\
60 x^{3}+y^{3}+z^{3} & =0
\end{aligned}
$$

Commentary. (1) This innocuous-sounding result lies quite deep. The fact that all its companions are of degree 3 may be misleading; there is, a priori, no guarantee that the companion curves to $V$ are isomorphic to smooth plane curves over $\mathbb{Q}$, and, in fact, there is a priori no upper bound to their degree in projective space. Establishing finiteness of $\mathscr{S}(C)$, something we could not even begin to do before the work of Rubin and Kolyvagin, is tantamount to establishing an upper bound to their degree.

(2) All five equations on this list have nontrivial rational solutions over $\mathbb{Q}_{p}$ for all prime numbers $p$ and over $\mathbb{R}$. The first four equations on the list possess no nontrivial rational solutions. The fifth equation possesses a nontrivial rational solution $(0,1,-1)$, and this solution is unique up to scalar multiplication (cf. [Ca2, §18]). If we take this point as "origin" of the projective curve $E$ defined by the equation

$$
60 x^{3}+y^{3}=z^{3}=0,
$$

\footnotetext{
${ }^{22}$ These are all of genus 1 ; smooth curves of degree $d$ in the projective plane are of genus $g=(d-1)(d-2) / 2$.
} 
then $E$ is an elliptic curve over $\mathbb{Q}$ isomorphic to the jacobian of all five curves on the list.

(3) In analogy with the discussion in $\S 2$ of the Hasse-Minkowski Theorem, giving the full list of companions as we have just done does not complete in a satisfactory manner the discussion of passage from local to global for this example: the demanding reader might ask how, if we are given some projective smooth curve over $\mathbb{Q}$ together with the data that exhibits it as a companion to Selmer's $C$, we may find a specific isomorphism over $\mathbb{Q}$ between it and one of the members of our list. ${ }^{23}$

In view of this type of example, why not weaken somewhat the local-to-global principle? Say that the local-to-global principle holds, up to finite obstruction for a class of varieties $\mathscr{V}$, if each member $V$ of $\mathscr{V}$ has only a finite number of nonisomorphic companions, i.e., if $\mathscr{S}(V)$ is a finite set for all $V$ of $\mathscr{V}$.

Despite the fact that we now, thanks to Rubin and Kolyvagin, can produce examples of some curves of genus 1 for which the local-to-global principle holds up to finite obstruction, this question has not yet been resolved for all curves of genus 1 . In contrast, consider:

Curves of genus $\geq 2$. Here the local-to-global principle holds up to finite obstruction; moreover, this is a rather easy result (see the discussion in $\S 5$ and Part III).

Summary. The one outstanding unresolved case remaining for curves is the case of genus 1 .

\section{General projective varieties.}

Conjecture 1. The local-to-global principle holds, up to finite obstruction for all (smooth) projective varieties; that is, any projective variety over $\mathbb{Q}$ has only a finite number of nonisomorphic companions.

Notes. As will be discussed in Part III, the set $\mathscr{S}(V)$, of companions to $V$, depends only upon the nature of the $\operatorname{Gal}(\overline{\mathbb{Q}} / \mathbb{Q})$-module of automorphisms of $V / \overline{\mathbb{Q}}$, where $\overline{\mathbb{Q}}$ is an algebraic closure of $\mathbb{Q}$. For a general projective variety, this $\mathrm{Gal}(\overline{\mathbb{Q}} / \mathbb{Q})$-module consists of the $\overline{\mathbb{Q}}$-rational points of a "locally algebraic group" $\underline{\operatorname{Aut}}(V)$.

In specific instances when the group $\underline{\operatorname{Aut}}(V)$ is easy to deal with, we can prove Conjecture 1. For example, the proof of Conjecture 1 is particularly easy if $\underline{\operatorname{Aut}}(V)$ is finite. This accounts for the fact that we know the local-toglobal principle up to finite obstruction for curves of genus $\geq 2$. For the same reason $^{24}$, we know the local-to-global principle up to finite obstruction for all

\footnotetext{
${ }^{23}$ I am thankful for e-mail correspondence with Rubin in which he has proposed a direct and succinct recipe for this. To put it in slightly different terms, suppose that you are given a curve of $C$ of genus 1 over $\mathbb{Q}$ as a projective variety in $\mathbf{P}^{N}$ and you are merely told that $C$ has a rational point, but you are also given a basis of the Mordell-Weil group of its jacobian $E$. Here is how you can actually find a rational point on $C$. You first find any algebraic point $P$ on $C$ of some degree $d$. Then find an algebraic point $\tau$ on $E$ such that $d \cdot \tau$ is the class of the divisor (of degree 0 ) on $C: \Sigma P^{\sigma}-d \cdot P$, where the summation is over all $\mathbb{Q}$-conjugates of $P$. Let $\mathscr{E}$ denote a representative system for the group of points on $E$ whose $d$ th multiple lies in $E(\mathbb{Q})$, modulo $E(\mathbb{Q})$. Then $\mathscr{E}$ is a finite set of points of $E$. Running through the (finite) set of translates of $P$ by $e+\tau$ where $e$ is drawn from $\mathscr{E}$, you are guaranteed that one of these is $\mathbb{Q}$-rational.

${ }^{24} \mathrm{Cf}$. [KO].
} 
varieties $V / \mathbb{Q}$ of general type. ${ }^{25}$ For almost the same reason the local-to-global principle up to finite obstruction holds for all smooth hypersurfaces of dimension $\geq 3$, or more generally, for all smooth complete intersections of dimension $\geq 3$ (see Part III, §17, Corollary 4).

A question, which arose in a conversation with $\mathrm{N}$. Katz on these matters, is the following:

Is it within the range of the present "state-of-the-art" to show finiteness of the part of $\mathscr{S}(V)$ represented by projective varieties whose numerical invariants are "limited" in various ways? For example, fixing an integer $d$ define $\mathscr{S}(V)_{d}$ to be the subset of $\mathscr{S}(V)$ represented by projective varieties which are companions to $V$ and which admit a projective imbedding of degree $\leq d$. Is $\mathscr{S}(V)_{d}$ finite for every $d$ ?

6. The Tate-Shafarevich group. In this section, we will be considering abelian varieties over $\mathbb{Q}$; i.e., smooth, geometrically connected, projective varieties over $\mathbb{Q}$ which are also algebraic groups, the group law $A \times A \rightarrow A$ defined over $\mathbb{Q}$. Such algebraic groups are necessarily abelian; since $A(\mathbb{C})$, the complex points of $A$, form a connected compact and commutative Lie group, $A(\mathbb{C})$ is isomorphic, as a topological group, to a product of circles.

If $A / \mathbb{Q}$ is an abelian variety, then its Tate-Shafarevich group, denoted $\mathrm{III}(A / \mathbb{Q})$, may be defined, first as a set, to be the set of isomorphism classes over $\mathbb{Q}$ of companions of $A$ endowed with principal homogeneous group action by $A$. More precisely, $\mathrm{II}(A / \mathbb{Q})$ is the set of isomorphism classes over $\mathbb{Q}$ of pairs $(W, \alpha)$ where $W$ is a projective variety defined over $\mathbb{Q}$ which is a companion of $A$ and $\alpha: A \times W \rightarrow W$ is a mapping of projective varieties, defined over $\mathbb{Q}$, which is a principal homogeneous group action of $A$ on $W$. (Note. To guarantee that the action $\alpha$ is a principal homogeneous action, one need only check that it induces a principal homogeneous action of the Lie group $A(\mathbb{C})$ on the topological space $W(\mathbb{C})$.

The set $\mathrm{II}(A / \mathbb{Q})$ is given a (commutative) group structure via the natural Baer-sum construction for principal homogeneous spaces. ${ }^{26}$ Forgetting the prin-

\footnotetext{
${ }^{25}$ General type. Let $r$ be a positive integer. Consider global tensors $\tau$ on a (smooth) $n$ dimensional variety $V$ which for any system of local coordinates $z_{1}, \ldots, z_{n}$ can be expressed as $h(z)\left(d z_{1} \wedge d z_{2} \wedge \cdots \wedge d z_{n}\right)^{\otimes r}$ where $h$ is some holomorphic function. If, for some choice of $r$, there are "enough" linearly independent such tensors $\tau_{0}, \ldots, \tau_{\nu}$-enough in the sense that the rule which sends a point $v \in V$ to the point in $\nu$-dimensional projective space with homogeneous coordinates $\left[\tau_{0}(v), \ldots, \tau_{\nu}(v)\right]$ is almost everywhere defined and gives a birational imbedding of $V$ to $\nu$-dimensional projective space-then one says that $V$ is of general type. The adjective general signifies that, by some reckonings, many varieties have this property. For example, for (smooth) curves, "general type" is equivalent to genus $>1$; for surfaces, the varieties that are not of general type are either rational, ruled, elliptic surfaces, abelian surfaces, $K 3$ surfaces, or Enriques surfaces, for smooth hypersurfaces $V$ of degree $d$ in projective $\nu$-space, if $d \geq \nu+2$ then $V$ is of general type.

${ }^{26} \mathrm{Baer}-s u m$. If $W_{1}$ and $W_{2}$ are two principal homogeneous spaces for $A$, let $A$ act on $W_{1} \times W_{2}$ by the antidiagonal action, i.e., the action of $a$ in $A$ on $\left(w_{1}, w_{2}\right)$ is $\left(w_{1}+a, w_{2}-a\right)$, where we have written the action of $A$ on $W_{1}$ and on $W_{2}$ additively. Then the Baer-sum of the principle homogeneous spaces $W_{1}$ and $W_{2}$ is the quotient variety of $W_{1} \times W_{2}$ with respect to the antidiagonal action, this quotient variety being viewed as principle homogeneous space for $A$ by an action induced from the diagonal action of $A$ on $W_{1} \times W_{2}$.
} 
cipal homogeneous action $(W, \alpha) \mapsto W$ gives a natural map (of pointed sets)

$$
\amalg(A / \mathbb{Q}) \rightarrow \mathscr{S}(A / \mathbb{Q}) .
$$

For an analysis of this mapping, see Part III, $\S 18$, Theorem 5. In particular, in $\S 17$ we will see that there are a finite set $\mathscr{A}$ of abelian varieties $A^{\prime} / \mathbb{Q}$ and natural mappings

$$
\mathrm{II}\left(A^{\prime} / \mathbb{Q}\right) \rightarrow \mathscr{S}(A / \mathbb{Q})
$$

for each $A^{\prime} \in \mathscr{A}$ such that the images of these mappings produce a partition of the set $\mathscr{S}(A / \mathbb{Q})$ indexed by $\mathscr{A}$ and the image of $\amalg\left(A^{\prime} / \mathbb{Q}\right)$ in $\mathscr{S}(A / \mathbb{Q})$ may be identified with the orbit-space of $\mathrm{m}\left(A^{\prime} / \mathbb{Q}\right)$ under the natural action of the group of $K$-automorphisms of $A^{\prime}$. From this description (cf. explicitly, Part III, $\S 18$, Corollary 2 to Theorem 5 ) one sees that Conjecture 1 restricted to the class of abelian varieties over $\mathbb{Q}$ is equivalent to

Conjecture 2 (Tate-Shafarevich). For any abelian variety $A$ over $\mathbb{Q}, \amalg(A / \mathbb{Q})$ is finite.

The relation between $\amalg(A / \mathbb{Q})$ and $\mathscr{S}(A / \mathbb{Q})$ has a resonance, in the classical theory of Gauss and Lagrange, in the distinction between the problems of classifying integral binary quadratic forms up to strict equivalence and equivalence. The notion of equivalence of integral binary quadratic forms is more intuitive than and is historically prior to the notion of strict equivalence, but only with the latter notion does one get a natural group structure on classes of forms.

What is known, in general, about the group structure of $\amalg(A / \mathbb{Q})$ is that it a torsion abelian group with the property that the kernel of the homomorphism given by multiplication by any nonzero integer $n$ on it is finite.

7. Implications of the Tate-Shafarevich Conjecture in the direction of the general local-to-global principle (up to finite obstruction). In preparing this article I have been tantalized by the urge to show that the Tate-Shafarevich Conjecture (i.e., Conjecture 2 for abelian varieties over $\mathbb{Q}$ ) is equivalent to the local-toglobal principle up to finite obstruction (i.e., Conjecture 1) for all projective varieties over $\mathbb{Q}$. This I have not done, but after some conversations and correspondence with Yevsey Nisnevich and with Ofer Gabber, Theorem 2 has emerged. For its proof, see Part III, $\S 17$, Corollary 2 to Theorem 4 . Before we state the result, however, we must talk a bit about the group of connected components of automorphism groups.

Let $V / \mathbb{Q}$ be an arbitrary projective variety and $\underline{\operatorname{Aut}}(V)$ its locally algebraic group of automorphisms. Let us denote by $\Gamma(V)$ the group of connected components of the locally algebraic group $\underline{\operatorname{Aut}}(V) / \overline{\mathbb{Q}}$, endowed with its natural continuous $\operatorname{Gal}(\overline{\mathbb{Q}} / \mathbb{Q})$-action. Let $\Delta(V)$ denote the quotient of $\operatorname{Gal}(\overline{\mathbb{Q}} / \mathbb{Q})$ which acts faithfully on $\Gamma(V)$, and let $\Gamma \rtimes \Delta$ denote the semidirect product constructed via this action.

What is known about the structure of $\Gamma(V)$ ? As will be discussed in Part III, there is a finitely generated abelian group $N S(V)$ with a continuous $\operatorname{Gal}(\overline{\mathbb{Q}} / \mathbb{Q})$ action on which the group $\Gamma(V)$ acts with finite kernel (and in a manner compatible with its natural $\mathrm{Gal}(\overline{\mathbb{Q}} / \mathbb{Q})$-action). In particular, a quotient of $\Gamma(V)$ by a finite group is isomorphic to a subgroup of $\operatorname{GL}(n, \mathbb{Z})$ for some positive 
integer $n$, and therefore there is an upper bound to the orders of finite subgroups of $\Gamma(V)$. It seems that little else is known about the structure of $\Gamma(V)$ ! Is $\Gamma(V)$ finitely generated? Is it finitely presented? Is it an arithmetic group? Conversely, what arithmetic groups occur as $\Gamma(V)$ 's?

One can show (Part III, §17, Corollary 2):

Theorem 2. Let $V / \mathbb{Q}$ be a projective variety. Suppose that:

(a) the group $\Gamma(V)$ is finitely presented;

(b) there are only a finite number of distinct $\Gamma$-conjugacy classes of finite subgroups in the semidirect product $\Gamma \rtimes \Delta$ (i.e., the group $\Gamma(V)$ endowed with $\operatorname{Gal}(\overline{\mathbb{Q}} / \mathbb{Q})$-action is decent in the sense defined in Part III, §16); and

(c) the Tate-Shafarevich Conjecture holds ${ }^{27}$.

Then $\mathscr{S}(V / \mathbb{Q})$ is finite (i.e., Conjecture 1 holds for $V$ ).

Some notes. If $\Gamma(V)$ is finitely generated, then $\Delta(V)$ is finite. Ofer Gabber has sketched a proof that the conclusion of Theorem 2 holds even if one weakens conditions (a) to the requirement that $\Gamma(V)$ be finitely generated. Are there any projective varieties $V$ for which $\Gamma(V)$ does not satisfy (a) and (b), i.e., for which $\Gamma(V)$ is not decent? ${ }^{28}$

8. Examples of finiteness of the Tate-Shafarevich group. Let $E / \mathbb{Q}$ be a modular elliptic curve. That is, $E$ is an elliptic curve, defined over $\mathbb{Q}$, whose underlying Riemann surface admits a nonconstant holomorphic mapping from a compactification of the quotient of the upper half plane under the action of a congruence subgroup of $\operatorname{SL}(2, \mathbb{Z}) .^{29}$ The conjecture of Taniyama-Weil asserts that every elliptic curve over $\mathbb{Q}$ is modular.

We must now introduce the $L$-function of $E / \mathbb{Q}$. For most of our purposes, we may deal with a crude version of it, which we will call $L_{S}(E, s)$, where, if we have fixed a model

$$
y^{2}=x^{3}+a x+b
$$

for $E$ over $\mathbb{Q}$, with $a, b \in \mathbb{Z}$, the subscript $S$ will be any finite set of prime numbers which includes all the prime numbers dividing the discriminant of this model. If $p$ is a prime number not in $S$, define the local factor

$$
L_{p}(E, s):=\left(1-a_{p} p^{-s}++p^{1-2 s}\right)^{-1},
$$

where $a_{p}$ is the integer defined by the formula $1+p-a_{p}=$ the number of rational points of the projective curve (over the prime field $\mathbb{F}_{p}$ ) determined by reducing the above model modulo $p$. Then $L_{S}(E, s)$ is defined (initially) as the Dirichlet series

$$
\prod_{p \notin S} L_{p}(E, s)
$$

\footnotetext{
${ }^{27} \mathrm{Or}$, somewhat more specifically, that it holds for all twists of certain subabelian varieties of $\operatorname{Pic}^{0}(V)$ over $\mathbb{Q}$.

${ }^{28}$ Is it true that the automorphism groups of $K 3$ surfaces, for example, are decent? They are finitely generated. Are they arithmetic? I have begun to pester experts about this. Relevant here are [P-S-S, $\S 7$, Proposition and Theorem 1] which give somewhat explicit descriptions of the automorphism groups of $K 3$ surfaces: If $V$ is a $K 3$ surface over $\overline{\mathbb{Q}}, N S$ its Néron-Severi lattice, and $\Gamma$ its automorphism group, then $\Gamma$ is commensurate with $O(N S) / W(N S)$ where $O(N S)$ is the orthogonal group of the lattice $N S$, and $W(N S)$ is the subgroup generated by reflections coming from elements in $N S$ of square -2 . For a finer study of which $\Gamma$ 's occur, see Nikulin [Nik1, 2].

${ }^{29}$ This is, in fact, equivalent to the more usual formulation of "modularity", see [Ma2].
} 
where $p$ runs through all prime numbers not in $S$. Using either elementary, or not so elementary, estimates on the size of the $a_{p}$, one sees that the Dirichlet series $L_{S}(E, s)$ converges to yield an analytic function of $s$ in a right half plane. Under the hypothesis that $E$ is modular, this analytic function $L_{S}(E, s)$ extends to an entire function in the $s$-plane.

Remark. There is, in fact, a "good way" of extending the definition of the local factors $L_{p}(E, s)$ for prime numbers $p$ in $S$. The $L_{p}(E, S)$ are polynomials in $p^{-s}$ of degree $\leq 2$ (cf. [Ta]) explicitly given in terms of a "minimal model" for the elliptic curve $E$ over $\mathbb{Z}$, and the "good" $L$ function $L(E, s)$ is defined to be the product of these local factors $L_{p}(E, s)$ for all prime numbers $p$.

The methods of Kolyvagin, aided by results of Gross and Zagier, Waldspurger, Bump, Friedberg, and Hoffstein, and R. Murty and K. Murty, yield the following extraordinary result (which is an important piece of the Conjecture of Birch and Swinnerton and Dyer).

Theorem 3. If $E$ is a modular elliptic curve for which the Hasse-Weil L function $L_{S}(E, s)$ either does not vanish at the point $s=1$ or has a simple zero at $s=1$, then $\mathrm{II}(E / \mathbb{Q})$ is finite.

In the former case, i.e., if $L_{S}(E, 1) \neq 0$, then $E$ possesses only a finite number of $\mathbb{Q}$-rational points. If $L_{S}(E, s)$ has a simple zero, then the group $E(\mathbb{Q})$ of $\mathbb{Q}$-rational points of $E$ is of rank 1 .

The order of zero of $L_{S}(E, s)$ at $s=1$ is independent of the choice of set of primes $S$ and would be the same if we dealt with the "good" $L$ function $L(E, s)$. For refinements of the above theorem, see [Coa, K1, K2, R1-8].

9. Returning to Selmer's curve. Briefly, this is what goes into the proof of Theorem 1: the last of the five curves in the list (given in the statement of Theorem 1) has a $\mathbb{Q}$-rational point and can be identified with the jacobian, call it $E$, of all five curves (cf. [Ca2, Chapters 18,20$]$ where it is shown that the cubic curve $a x^{3}+b y^{3}+c z^{3}=0$, for nonzero rational numbers $a, b, c$ has, as jacobian, the elliptic curve $\left.a b c x^{3}+y^{3}+z^{3}=0\right)$. This elliptic curve $E$ has complex multiplication, and a computation gives that the ratio $L(E, 1) / \Omega$ is equal to 9 , where $L(E, s)$ is the (good) $L$-function of $E$ and $\Omega$ is the real period of $E$. It follows then from the recent work of Rubin [R5] that, if $\mathrm{III}=\mathrm{II}(E / \mathbb{Q})$ is the Tate-Shafarevich group of $E$ over $\mathbb{Q}$, then III is finite and of order equal to a power of 2 times a power of 3 . Checking prior computations of the 2- and 3-primary components of III in tables of Stephens [St], one then has that III is a product of two cyclic groups of order 3. By Part III, $\S 18$, Corollary 1 to Theorem 5 the set of companions $\mathscr{S}(C)$ may be identified with the quotient-set of III under the involution $x \mapsto-x$ and, therefore, has cardinality $=1+(9-1) / 2=5$. One is left with the chore of finding representatives for the 5 companions; this being done by the list presented in the statement of the theorem, it remains only to check that all five curves on our list possess rational points over $\mathbb{Q}_{p}$ for all prime numbers $p$, and over $\mathbb{R}$, which they do, and that they are pairwise nonisomorphic over $\mathbb{Q}$, which they are $^{30}$.

\footnotetext{
${ }^{30}$ Here are some hints about this latter assertion. Let $E[3]$ be the kernel of multiplication by 3 in $E$, and note that the natural mapping $H^{1}(\mathbb{Q}, E[3]) \rightarrow H^{1}(\mathbb{Q}, E)$ is injective (since $E(\mathbb{Q})$
} 


\section{PART II}

10. The Tate-Shafarevich group and the Selmer group. Let $\overline{\mathbb{Q}}$ be the algebraic closure of $\mathbb{Q}$ in $\mathbb{C}$, and for each prime number $p$ fix an embedding of $\overline{\mathbb{Q}}$ in $\overline{\mathbb{Q}}_{p}$, an algebraic closure of $\mathbb{Q}_{p}$. Let $G$ denote the Galois group $\operatorname{Gal}(\overline{\mathbb{Q}} / \mathbb{Q})$, and for each $p$ let $G_{p}:=\operatorname{Gal}\left(\overline{\mathbb{Q}}_{p} / \mathbb{Q}_{p}\right)$ and $G_{\infty}:=\operatorname{Gal}(\mathbb{C} / \mathbb{R})$, these being viewed as closed subgroups of $G$ (after our choices of embeddings).

Fix an elliptic curve $E / \mathbb{Q}$. If $K$ is a field extension of $\mathbb{Q}$, the group of $K$-rational points of $E$ is denoted $E(K)$. Since $E$ is defined over $\mathbb{Q}$, there is a natural (continuous) action of $G$ on $E(\overline{\mathbb{Q}})$, of $G_{p}$ on $E\left(\overline{\mathbb{Q}}_{p}\right)$, and of $G_{\infty}$ on $E(\mathbb{C})$. The cohomological definition of $\mathbb{\amalg}(E / \mathbb{Q})$ is as follows.

$$
\mathrm{III}=\mathrm{\amalg}(E / \mathbb{Q}):=\bigcap \operatorname{ker}\left\{H^{1}(G, E(\overline{\mathbb{Q}})) \rightarrow H^{1}\left(G_{p}, E\left(\overline{\mathbb{Q}}_{p}\right)\right)\right\}
$$

where the intersection is taken over all "finite" primes $p$ and $p=\infty$. It is useful to lighten the notation somewhat and to convene that

$$
\begin{aligned}
H^{1}(G, E) & :=H^{1}(G, E(\overline{\mathbb{Q}})), \\
H^{1}\left(G_{p}, E\right) & :=H^{1}\left(G_{p}, E\left(\overline{\mathbb{Q}}_{p}\right)\right), \\
H^{1}\left(G_{\infty}, E\right) & :=H^{1}\left(G_{\infty}, E(\mathbb{C})\right) .
\end{aligned}
$$

If $A$ is an abelian group, and $n$ an integer, let $A[n]$ denote the kernel of multiplication by $n$, so that we have the "Kummer" exact sequences of continuous $G$-modules,

$$
0 \rightarrow E(\overline{\mathbb{Q}})[n] \rightarrow E(\overline{\mathbb{Q}}) \stackrel{n}{\rightarrow} E(\overline{\mathbb{Q}}) \rightarrow 0,
$$

and of continuous $G_{p}$-modules,

$$
0 \rightarrow E\left(\overline{\mathbb{Q}}_{p}\right)[n] \rightarrow E\left(\overline{\mathbb{Q}}_{p}\right) \stackrel{n}{\rightarrow} E\left(\overline{\mathbb{Q}}_{p}\right) \rightarrow 0,
$$

each yielding long exact sequences on cohomology, pieces of which, following our convention, can be written as the horizontal exact sequences occurring in the commutative diagram:

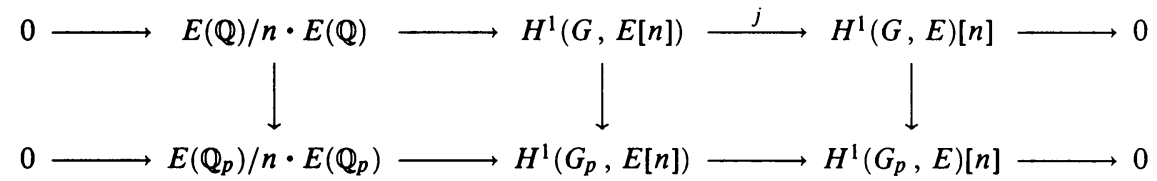

Visibly, $\mathrm{II}[n]$, the kernel of multiplication by $n$ in $\mathrm{III}$, is the intersection for all $p \leq \infty$ of the kernels of the right-hand vertical arrows in the above diagrams (for all $p$ ).

Definition. The $n$-Selmer group (of the elliptic curve $E / \mathbb{Q}$ ), denoted $S[n]$, is the subgroup of $H^{1}(G, E[n])$ defined as the full inverse image of $\amalg[n] \subset$ $H^{1}(G, E)[n]$ under the map labelled $j$ above.

For any finite field extension $K$ of $\mathbb{Q}$ in $\overline{\mathbb{Q}}$, making the base change from $\mathbb{Q}$ to $K$, we have the Tate Shafarevich group of $E / K$ and the analogously defined

vanishes). Now if $C$ is any one of our five curves $a x^{3}+b y^{3}+c z^{3}$, let $\beta, \gamma \in \overline{\mathbb{Q}}$ be such that $\beta^{3}=b, \gamma^{3}=c$. The cohomology class corresponding to $C$ in $H^{1}(\mathbb{Q}, E)$ is the image of the class in $H^{1}(\mathbb{Q}, E[3])$ represented by the 1 -cocycle which sends an element $\sigma \in \mathrm{Gal}(\overline{\mathbb{Q}} / \mathbb{Q})$ to the point $\left(0,1,(\beta / \gamma)^{\sigma-1}\right)$ in $E[3]$. These 1-cocycles lie in distinct cohomology classes, for our five curves $C$. 
$n$-Selmer group of $E / K$, which we will denote $\amalg_{K}$ and $S_{K}[n]$, respectively. Essentially by definition these fit into an exact sequence

$$
0 \rightarrow E(K) / n \cdot E(K) \rightarrow S_{K}[n] \rightarrow \mathrm{W}_{K}[n] \rightarrow 0 .
$$

The importance of the Selmer group is that, as the above exact sequence displays, it packages information concerning both the Mordell-Weil group and the Tate-Shafarevich group; but the Selmer group is, in many instances, curiously more tractable than either of them. This is rather like the phenomenon met in the study of number fields, where, at times, the product of class number times regulator is more readily computed than either factor. In any event, to study elements of order $n$ in $\amalg_{K}$, we pass to the study of elements

$$
s \in S_{K}[n] \subset H^{1}\left(G_{K}, E[n]\right),
$$

where $G_{K}$ is the closed subgroup of $G$ which is the identity on $K$.

11. Local control of the Selmer group. At this point we are ready to discuss a step in Kolyvagin's program that may seem modest but is of crucial importance in setting the stage for what is to come. The "abstract" group $E[n]$ is isomorphic to a product of two cyclic groups of order $n$ and consequently its full automorphism group is isomorphic to the finite group $\mathrm{GL}_{2}(\mathbf{Z} / n \mathbf{Z})$. The natural action of $G_{K}$ on $E[n]$ gives us, up to equivalence, a representation $G_{K} \rightarrow \mathrm{GL}_{2}(\mathbb{Z} / n \mathbb{Z})$, whose kernel we will denote $G_{M}$, and, to be consistent, we use the letter $M$ to denote the finite extension field of $K$ in $\overline{\mathbb{Q}}$ comprised of the elements in $\overline{\mathbb{Q}}$ fixed by $G_{M}$. So, letting $\Delta_{n}$ denote $\operatorname{Gal}(M / K)=G_{K} / G_{M}$, we have the natural inclusion $\Delta_{n} \subset \mathrm{GL}_{2}(\mathbb{Z} / n \mathbb{Z})$.

Let us now say that the integer $n$ is good if the "restriction mapping" on cohomology, described below, is injective:

$$
H^{1}\left(G_{K}, E[n]\right) \rightarrow H^{1}\left(G_{M}, E[n]\right)^{\Delta_{n}}=\operatorname{Hom}_{\Delta_{n}}\left(G_{M}, E[n]\right) .
$$

The usefulness of this definition of goodness is that:

(a) invoking the known richness of action of the Galois groups of number fields on $n$-torsion in elliptic curves, the hypothesis of goodness is not a strong hypothesis on $n$ (and even when $n$ is not good, the kernel of the "restriction mapping", for $n$ replaced by powers of $n$, is finite, of order bounded independent of the power).

(b) any element $h \in H^{1}\left(G_{K}, E[n]\right)$ (and consequently, also, any element $s$ in the Selmer group) gives rise, by the "restriction homomorphism" above, to a continuous $\Delta_{n}$-equivariant homomorphism; if $n$ is good, then triviality of the $\Delta_{n}$-equivariant homomorphism $h: G_{M} \rightarrow E[n]$ implies triviality of the element $h \in H^{1}\left(G_{K}, E[n]\right)$ giving rise to it.

From now on we assume $n$ to be good, and we will freely identify elements of $H^{1}\left(G_{K}, E[n]\right)$ with the $\Delta_{n}$-equivariant homomorphisms $G_{M} \rightarrow E[n]$ to which they give rise. This allows us to "study" elements $s \in S_{K}[n]$ by local means, in the following sense.

Let $v$ run through all places of $K$, both nonarchimedean and archimedean, and let $K_{v}$ denote the completion of $K$ at $v, \bar{K}_{v}$ an algebraic closure containing $\overline{\mathbb{Q}}$, and $G_{K_{v}}:=\operatorname{Gal}\left(\bar{K}_{v} / K_{v}\right)$. Then we have the exact sequence

$$
0 \rightarrow E\left(K_{v}\right) / n \cdot E\left(K_{v}\right) \rightarrow H^{1}\left(G_{K_{v}}, E[n]\right) \rightarrow H^{1}\left(G_{K_{v}}, E\right)[n] \rightarrow 0,
$$


and, directly from the definition of the Selmer group, we have that the image of an element $s \in S_{K}[n]$ under the natural homomorphism $H^{1}\left(G_{K}, E[n]\right) \rightarrow$ $H^{1}\left(G_{K_{v}}, E[n]\right)$ maps to an element, call it $s_{v}$, in the subgroup

$$
E\left(K_{v}\right) / n \cdot E\left(K_{v}\right) \subset H^{1}\left(G_{K_{v}}, E[n]\right) .
$$

Since $n$ is good, and since a homomorphism from $G_{K}$ is trivial if it is trivial on all decomposition groups, we have something that could be called the principle of local control:

If the "local" elements $s_{v} \in E\left(K_{v}\right) / n \cdot E\left(K_{v}\right)$ vanish for all places $v$, then $s$ vanishes.

12. Class field theory, duality, and Kolyvagin test classes. The $G_{K}$-module $E[n]$ admits a (nondegenerate) self-pairing, called the "Weil-pairing"

$$
E[n] \times E[n] \rightarrow \mu_{n}
$$

where $\mu_{n}$ is the $G_{K}$-module of $n$th roots of unity. The Weil pairing induces a pairing on local cohomology

$$
\begin{aligned}
H^{1}\left(G_{K_{v}}, E[n]\right) \times H^{1}\left(G_{K_{v}}, E[n]\right) & \rightarrow H^{2}\left(G_{K_{v}}, \mu_{n}\right) \subset \mathbb{Q} / \mathbb{Z} \\
(x, y) & \mapsto\langle x, y\rangle_{v}
\end{aligned}
$$

where the inclusion of $H^{2}\left(G_{K_{v}}, \mu_{n}\right)$ in $\mathbb{Q} / \mathbb{Z}$ is given by Local Class Field Theory. Important for us will be the fact ${ }^{31}$ that if both $x$ and $y$ map to zero under the homomorphism to $H^{1}\left(G_{K_{v}}, E\right)$, then $\langle x, y\rangle_{v}=0$.

The Weil pairing also induces a pairing on global cohomology

$$
\begin{aligned}
H^{1}\left(G_{K}, E[n]\right) \times H^{1}\left(G_{K}, E[n]\right) & \rightarrow H^{2}\left(G_{K}, \mu_{n}\right) \subset \bigoplus_{v} H^{2}\left(G_{K_{v}}, \mu_{n}\right) \subset \bigoplus_{v} \mathbb{Q} / \mathbb{Z} \\
(x, y) & \mapsto \bigoplus\left\langle x_{v}, y_{v}\right\rangle_{v}
\end{aligned}
$$

where $x_{v}$ and $y_{v}$ are the images of $x$ and $y$ in the local cohomology $H^{1}\left(G_{K_{v}}, E[n]\right)$, the direct sum on the right being taken over places $v$ of $K$. The inclusion on the right is that given by Global Class Field Theory, which embeds $H^{2}\left(G_{K}, \mu_{n}\right)$ into the kernel of the sum mapping $\bigoplus_{v} \mathbb{Q} / \mathbb{Z} \rightarrow \mathbb{Q} / \mathbb{Z}$.

Kolyvagin's basic strategy. With our data, $E, K, n$, fixed and understood, let $w$ be a place of $K$. By a Kolyvagin test class for $w$ let us mean a cohomology class $c \in H^{1}\left(G_{K}, E[n]\right)$, which has the property that $c$ goes to 0 under the composition of natural mappings $H^{1}\left(G_{K}, E[n]\right) \rightarrow H^{1}\left(G_{K_{v}}, E[n]\right) \rightarrow$ $H^{1}\left(G_{K_{v}}, E\right)$ for all places $v \neq w$ of $K$ and does not go to 0 for $v=w$.

Let us first see that any Kolyvagin test class imposes a local condition on every element of the Selmer group. For, if $s \in S_{K}[n]$ and $c$ is a Kolyvagin test class, we have that $\left\langle c_{v}, s_{v}\right\rangle_{v}=0$ for $v \neq w$, since for places different from $w$, both $c_{v}$ and $s_{v}$ map to zero in $H^{1}\left(G_{K_{v}}, E\right)$.

On the other hand, by the consequence of Global Class Field Theory cited above, we have

$$
\sum\left\langle c_{v}, s_{v}\right\rangle_{v}=0
$$

\footnotetext{
${ }^{31}$ One might add that the pairing $E\left(K_{v}\right) / n \cdot E\left(K_{v}\right) \times H^{1}\left(G_{K_{v}}, E\right)[n] \rightarrow \mathbb{Q} / \mathbb{Z}$ induced by the (Weil) self-pairing on $H^{1}\left(G_{K_{v}}, E[n]\right)$ is compatible, up to sign, with the pairing coming from Tate Local Duality (for abelian varieties). Cf. the related discussion [Mi4, Chapter I, §3, pp. 54-55].
} 
the summation being taken over all places $v$ of $K$.

Putting these together, we get a local condition at $w$, which is satisfied for any element $s$ in the Selmer group $S_{K}[n]$; namely,

$$
\left\langle c_{w}, s_{w}\right\rangle_{w}=0 \text {. }
$$

Kolyvagin's strategy, roughly put, is to produce a large and systematic collection of test classes $c$ so as to obtain enough conditions of the type (1) on the local components of elements $s$ to provide the tightest bounds on the size of the Selmer group.

For example, in one series of applications, $n$ is a prime number $>2$, the field $K$ is a quadratic imaginary field, and, letting the superscript ${ }^{ \pm}$denote \pm eigenspace for the nontrivial automorphism of $K$ (we are in a situation where each of the two eigenspaces $\left\{E\left(K_{w}\right) / n \cdot E\left(K_{w}\right)\right\}^{ \pm}$is cyclic of order $\left.n\right)$. In this setup, the mere existence of a Kolyvagin test class for $w$ in the \pm eigenspace guarantees (via condition (1)) that, for any Selmer class $s \in S_{K}[n]^{ \pm}$(same sign), we have $s_{w}=0$.

Naturally, one might (and in fact one does) wish, at times, to loosen the requirement on a test class $c$ that goes to 0 in $H^{1}\left(G_{K_{v}}, E\right)$ for all but one place $v$. Fixing a finite set $S$ of places, one can make similar good use of classes $c$ that go to 0 in $H^{1}\left(G_{K_{v}}, E\right)$ for all $v$ outside the finite set $S$.

13. Rational points in extension fields yielding "test classes". The basic mechanism that links rational points to Kolyvagin test classes is encapsulated by the diagram displayed below. Here, $L / K$ is a finite Galois extension with Galois group $\mathscr{G}$, and we assume that $L / K$ is unramified outside the single place $w$ of $K$ and that the "restriction mapping", labelled Res below, is an isomorphism:

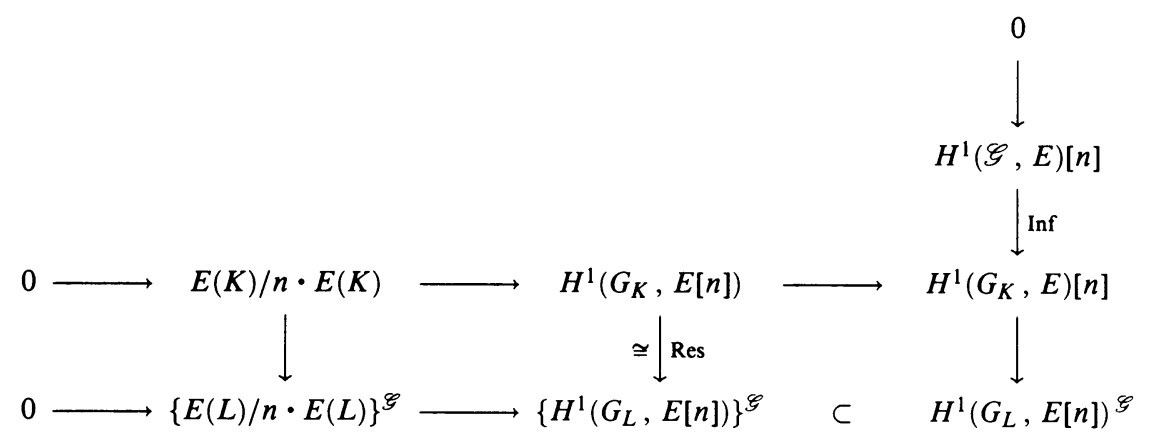

Now let $P \in E(L)$ be a rational point satisfying the following two conditions-the first being the "serious" condition, and the second a minor one:

(a) For all $\sigma \in \mathscr{G}, p^{\sigma}-P$ is divisible by $n$ in $E(L)$.

(b) For each place $\nu$ of $L$ which does not lie over the place $w$ of $K$, the specialization of $P$ to the group of connected components of the Neron fiber of $E$ at $\nu$ is of order relatively prime to $n$.

For Néron models, see Artin's exposition (in [A2]) of Néron's original paper [Né]; see also Grothendieck's Exp. IX in [Grot3] and [BLR].

Let us call rational points $P \in E(L)$ satisfying (a), (b) above Kolyvagin rational points.

By $(\mathrm{a}), P$ gives rise to a class $[P]$ in $\{E(L) / n \cdot E(L)\}^{\mathscr{G}}$. 
Definition. The class $c=c_{P} \in H^{1}\left(G_{K}, E[n]\right)$ is the unique homology class such that $\operatorname{Res}(c)$ is equal to the image of $[P]$ in $\left\{H^{1}\left(G_{L}, E[n]\right)\right\}^{\mathscr{G}}$.

There is a unique homology class $d \in H^{1}(\mathscr{G}, E)[n]$ such that $\operatorname{Inf}(d)$ is the image of $c$ in $H^{1}\left(G_{K}, E\right)[n]$. Using (b) one can show that the restriction of the class $d$ to decomposition groups attached to every place $v \neq w$ of $K$ vanishes, i.e., that $c_{P}$ is a Kolyvagin test class for $w$ provided its image in $H^{1}\left(G_{K_{w}}, E\right)$ does not vanish. Clearly a necessary requirement for this nonvanishing to occur is that $[P]$ be a "new" class in $\{E(L) / n \cdot E(L)\}^{\mathscr{G}}$, i.e., a class not in the image of $E(K) / n \cdot E(K)$.

Summary. We have started with an elliptic curve $E / \mathbb{Q}$. We have fixed a "good" nonzero integer $n$. We have somewhat silently passed to a finite extension field $K / \mathbb{Q}$. Let us call this $K$ the first auxiliary field extension: as already hinted, it will be chosen to be a suitable quadratic imaginary field extension of $\mathbb{Q}$. For any place $w$ of $K$, we now wish to find a suitable Galois extension field $L / K$ unramified outside $w$ (call $L / K$ the second auxiliary field extension) and also a "Kolyvagin" rational point $P$ in $E(L)$ which yields a "new" class $[P]$ in $\{E(L) / n \cdot E(L)\}^{\mathscr{G}}$.

The construction of a suitable "first" auxiliary field extension $K / \mathbb{Q}$ and then an ample and systematic supply of "second" auxiliary field extensions $L / K$ together with rational points $P$ is the punch line of Kolyvagin's program. For then we would have a significant supply of Kolyvagin test classes $c_{P}$ which would impose a significant number of conditions on the local components of elements of the $n$-Selmer group. By the "principle of local control", one then would have control of the $n$-Selmer group itself.

14. Heegner points. For any quadratic imaginary field extension $K / \mathbb{Q}$ (an appropriate one of these will be our choice of "first auxiliary field extension") and for any positive integer $f$, let $K_{f}$ denote the ring class field of conductor $f$ over $K$ (for suitable $f, K_{f} / K$ will be our choice of "second auxiliary field extension" $L / K)$.

We have a tower of field extensions, with Galois Groups as marked below, and where the full Galois group $\operatorname{Gal}\left(K_{f} / \mathbb{Q}\right)$ is equal to a "generalized dihedral group", i.e., an extension of a cyclic group of order 2 by the abelian group $\mathscr{G}_{f}:=\operatorname{Gal}\left(K_{f} / K\right)$, with action of the nontrivial element in the cyclic group on $\mathscr{G}_{f}$ given by multiplication by -1 .

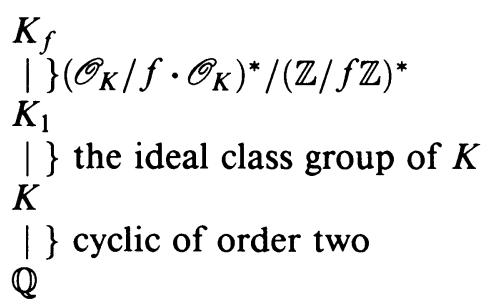

Here, $\mathscr{O}_{K}$ denotes the ring of integers of $K$. If $\mathscr{O}_{K, f}$ denotes the order $\mathbb{Z}+f \cdot \mathscr{O}_{K} \subset \mathscr{O}_{K}$, we have that $\operatorname{Gal}\left(K_{f} / K\right)$ is isomorphic to the group of isomorphism classes of locally free $\mathscr{O}_{K, f}$-modules of rank one.

As previously mentioned, the Taniyama-Weil conjecture asserts that any elliptic curve $E / \mathbb{Q}$ can be realized as a quotient curve (over $\mathbb{Q}$ ) of the modular curve $X_{0}(N)$ for a suitable positive integer $N$. Noncuspidal points $x$ of 
$X_{0}(N)$ may be "identified" with pairs $(\mathscr{E}, C)$ up to isomorphism, where $\mathscr{E}$ is an elliptic curve and $C$ is a cyclic subgroup of $\mathscr{E}$ of order $N$. The point $x$ is rational over a given field extension of $\mathbb{Q}$ if and only if the pair $(\mathscr{E}, C)$ is definable over that number field. Suppose then that we have a nonconstant mapping, defined over $\mathbb{Q}, \varphi: X_{0}(N) \rightarrow E$.

We now choose a quadratic imaginary field $K \subset \mathbb{C}$ such that all prime factors of $N$ split in $\mathscr{O}_{K}$. Under this hypothesis, there is an ideal $\mathscr{N} \subset \mathscr{O}_{K}$ such that $\mathscr{O}_{K} / \mathscr{N}$ is cyclic of order $N$; we choose such an ideal.

For any positive integer $f$ relatively prime to $N$, the ideal $\mathscr{N}_{f}:=\mathscr{N} \cap \mathscr{O}_{K, f}$ is an invertible $\mathscr{O}_{K, f}$-module such that $\mathscr{O}_{K, f} / \mathscr{N}_{f}$ is cyclic of order $N$. Let $\mathscr{E}_{f}$ be the elliptic curve $\mathbb{C} / \mathscr{O}_{K, f}$, and let $C_{f} \subset \mathscr{E}_{f}$ be the cyclic subgroup of order $N$ given by $\mathscr{N}_{f}^{-1} / \mathscr{O}_{K, f}$. Let $x_{K, f} \in X_{0}(N)$ be the point corresponding to the pair $\left(\mathscr{E}_{f}, C_{f}\right)$, and put $y_{K, f}=\varphi\left(x_{K, f}\right)$.

Then $y_{K, f}$ is a $K_{f}$-rational point of $E$. We refer to it as the Heegner point of $E$ (for the choices: $K \subset \mathbb{C}, \mathscr{N}, f$, and the parametrization $\varphi$ ). The collection of Heegner points for a fixed $K$ and all positive integers $f$ which are relatively prime to $N$ satisfy the axioms of what Kolyvagin calls a "Euler system", these axioms being the essence of what is needed to produce a supply of "Kolyvagin points".

To avoid getting at all into the technicalities of the passage from Heegner points to Kolyvagin points, and yet to give some small, but honest, hint of the flavor of it, consider the following:

We restrict attention to the case where $n$ is a prime number. Now choose $f$ to be a prime number which is inert (and unramified) in $K$, which does not divide $N \cdot n$, and such that the Frobenius element at $(f)$ in $G_{\mathbb{Q}}$ acts as a nonscalar involution on the group of $n$-torsion points of the elliptic curve $E$. Then $\operatorname{Gal}\left(K_{f} / K_{1}\right)$ is cyclic of order $f+1$. Fix a generator $\gamma \in \operatorname{Gal}\left(K_{f} / K_{1}\right) \subset$ $\operatorname{Gal}\left(K_{f} / K\right)$, and fix a representative system $S \subset \operatorname{Gal}\left(K_{f} / K\right)$ modulo $\operatorname{Gal}\left(K_{f} / K_{1}\right)$ so that every element of $\operatorname{Gal}\left(K_{f} / K\right)$ is in $\gamma^{i} \cdot S$ for a unique integer $i$ in the range $0 \leq i \leq f$. Put

$$
P_{K, f}:=\sum i \cdot \gamma^{i} \cdot s \cdot y_{K, f} \in E\left(K_{f}\right),
$$

the summation being taken over all $s \in S$ and $i$ in the above range.

Then (see [Gros] for a very neatly written proof of this) taking our "second auxiliary choice" $L / K$ to be $K_{f} / K$, the point $P_{K, f}$ is a "Kolyvagin point"; i.e., it satisfies (a), (b) above, and, moreover, the class $\left[P_{K, f}\right]$ in $\{E(L) / n E(L)\}^{\mathscr{G}}$ is independent of the choice of representative system $S$; it is independent up to scaling by $(\mathbb{Z} / n \mathbb{Z})^{*}$ of the choice of generator $\gamma$.

\section{PART III ${ }^{32}$}

15. The cohomology of locally algebraic group schemes. In Part I, for ease of notation, the base field was taken to be $\mathbb{Q}$, but here it is more natural to allow arbitrary number fields $K$ (of finite degree over $\mathbb{Q}$ ). For $v$ a place of $K$, we let $K_{v}$ denote the completion of $K$ with respect to $v$. So, if $V$ is a projective variety over $K$, we have the analogous definition of companion variety to $V$, i.e., a projective variety $V^{\prime}$ over $K$ which is isomorphic to $V$

\footnotetext{
${ }^{32}$ I am very thankful to Ofer Gabber and Yevsey Nisnevich for the crucial help they gave to me while I was writing this.
} 
over every completion $K_{v}$. We also have $\mathscr{S}(V / K)$, the set of isomorphism classes of companions (over $K$ ).

Let $X$ denote the spectrum of the ring of integers in $K$, and we reserve the letter $S$ for finite sets of places of $K$, containing all infinite places. Let $X-S$ be the open subscheme in $X$ which is the complement of the closed points corresponding to the nonarchimedean places of $S$.

We shall be studying group schemes over $X-S$ which are (separated and) locally of finite type but not necessarily of finite type. To signal this, we shall refer to such group schemes as locally algebraic group schemes (extending the terminology in [BS] where locally algebraic groups are over fields rather than over more general base schemes). We shall refer to a group scheme which is of finite type as an algebraic group scheme.

Let $G$, then, be a smooth locally algebraic group scheme over $X-S$. That is, $G$ is a group scheme (separated, and), locally of finite type over $X-S$, for which $G^{0}$, the connected component containing the identity section, is a smooth connected open subgroup scheme of $G$, of finite type over $X-S$. If $Z \rightarrow X-S$ is any morphism of schemes, the pullback of our group scheme $G$ to the base $Z$ is denoted $G / Z$. Whenever we write $H^{1}(Z, G)$ we can mean, equivalently, étale or flat (fppf) cohomology of $G / Z$ over $Z$ (compare [Grot]). Given a $G$-valued 1-cocycle $c$ for the étale topology on $Z$, we can use the conjugation action of $G$ on $G$ to "twist" the locally algebraic group scheme $G / Z$ by $c$ (an "inner twist"; $c f$. [Gi]) and the isomorphism class of the resulting locally algebraic group scheme over $Z$ is dependent only upon the cohomology class $\gamma$ in $H^{1}(Z, G)$ of our cocycle $c$; we shall denote this "inner twist" by $G_{\gamma} / Z$.

Since our groups are, in the main, noncommutative, we are referring here to noncommutative one-dimensional cohomology, given the structure only of pointed set. Giving the concepts " $\Pi$ " and "ker" the evident meanings in the context of pointed sets, we let

$$
\mathrm{\amalg}(G):=\operatorname{ker}\left\{H^{1}(K, G) \rightarrow \prod_{v} H^{1}\left(K_{v}, G\right)\right\},
$$

the product being taken over all places $v$ of $K$, and

$$
\amalg_{S}(G):=\operatorname{ker}\left\{H^{1}(X-S, G) \rightarrow \prod_{v \in S} H^{1}\left(K_{v}, G\right)\right\} .
$$

Examples. (1) by a "locally constant" group scheme $G / S$ we mean a group scheme which is locally constant for the étale topology, i.e., such that there is a finite étale cover $S^{\prime} \rightarrow S$ for which the pullback $G / S^{\prime}$ is a constant group scheme. Let $G / K$ be a locally constant group over the number field $K$, and let $L / K$ be the (minimal) finite splitting field for the action of $\operatorname{Gal}(\bar{K} / K)$ on $G(\bar{K})$. Then $\amalg(G)$ is isomorphic to

$$
\operatorname{ker}\left\{H^{1}(L / K, G) \rightarrow \prod_{v} H^{1}\left(K_{v}, G\right)\right\} .
$$

The reason for this is the following. First, if the action of $\operatorname{Gal}(\bar{K} / K)$ on $G(\bar{K})$ is trivial, then $\amalg(G)$ is trivial, because in that case $H^{1}(K, G)$ is identified with conjugacy classes of continuous homomorphisms from $\operatorname{Gal}(\bar{K} / K)$ 
to $G(\bar{K})$, and if a homomorphism restricts to the trivial homomorphism from $\operatorname{Gal}\left(\bar{K}_{v} / K_{v}\right)$ to $G\left(\bar{K}_{v}\right)$, for all places $v$, then it is trivial. In the general situation, then, an element of $\mathrm{m}(G) \in H^{1}(K, G)$ must map to the trivial element in $H^{1}(L, G)$ and therefore come from $H^{1}(L / K, G)$, as stated.

Corollary. Let $G$ be a locally constant group whose Galois action is split by a finite cyclic extension of $K$. Then $\mathrm{II}(G)$ is trivial.

Proof. Let $L / K$ be the finite cyclic extension which splits the Galois action of $G$. By the Cebotarev Theorem, there is a place $w$ of $L$ lying over a place $v$ of $K$ (which is unramified, and) for which $\operatorname{Gal}\left(L_{w} / K_{v}\right)=\operatorname{Gal}(L / K)$. It then follows that for this $v$, the mapping $H^{1}(L / K, G) \rightarrow H^{1}\left(K_{v}, G\right)$ is injective.

(2) Let $G$ be a locally algebraic group over $K$ which is an extension ${ }^{33}$ of a locally constant group which is an arithmetic group (whose continuous $\mathrm{Gal}(\bar{K} / K)$ action comes from an action of an ambient linear algebraic group over $\mathbb{Q}$ ) by a linear algebraic group (such groups are said to be of "type ALA" in [BS]). Then $\mathrm{III}(G)$ is finite and, moreover, the mapping

$$
H^{1}(K, G) \rightarrow \prod_{v} H^{1}\left(K_{v}, G\right)
$$

is proper, i.e., has finite fibers [BS, Theorem 7.1].

If $G$ is a connected, simply connected, semisimple algebraic group over $K$, then this mapping is now known to be an isomorphism (if one either includes or neglects to include the factors on the right corresponding to nonarchimedean $v$, since $H^{1}\left(K_{v}, G\right)=0$ for finite $v$, and connected, simply connected, semisimple algebraic groups $G$ ). This fact, going under the heading The Hasse Principle for connected, simply connected, semisimple algebraic groups, has been the fruit of a long development (cf. [Se2, Kne] for a discussion of the work on this problem for the classical semi-simple groups; Harder's paper [Hard] for a proof of the above assertion for all connected, simply connected, semisimple algebraic groups $G / K$ which do not have a factor of type $E_{8}$; Platonov's survey [Pl] for a neat discussion of where the problem stood circa 1982; and Chernousov's resolution of what remained to be done with respect to $E_{8}[\mathrm{Ch}]$, thereby finishing the problem).

16. Discrete locally algebraic group schemes. Fix $\bar{K}$ an algebraic closure of $K$, and consider the category of discrete $\mathrm{Gal}(\bar{K} / K)$-groups, i.e., "abstract groups" (viewed as discrete topological groups) endowed with continuous $\mathrm{Gal}(\bar{K} / K)$ action. This category is equivalent to the category of discrete locally algebraic groups over $K$, the equivalence

$$
\text { discrete loc alg gp } \mathscr{G} / K \rightarrow \operatorname{discrete} \operatorname{Gal}(\bar{K} / K) \text {-gp } \Gamma
$$

being given by taking $\Gamma$ to be the group $\mathscr{G}(\bar{K})$ with its natural $\mathrm{Gal}(\bar{K} / K)$ Galois action. The discrete locally algebraic group $\mathscr{G} / K$ is locally constant (in the sense discussed in the previous section, i.e., locally constant for the étale topology) if and only if the action of $\operatorname{Gal}(\bar{K} / K)$ on $\Gamma$ factors through a finite quotient group (this being automatic if $\Gamma$ is finitely generated).

\footnotetext{
${ }^{33}$ We keep to the convention that a group $G$ is an extension of $A$ by $B$ if $B$ is the normal subgroup of $G$ and $A$ is the quotient group $G / B$.
} 
Let $\Gamma$ be a discrete $\operatorname{Gal}(\bar{K} / K)$-group. Denote by $\Delta=\operatorname{Gal}(L / K)$ the quotient of $\mathrm{Gal}(\bar{K} / K)$ which acts faithfully on $\Gamma$; i.e., $L \subset \bar{K}$ is the splitting field of the action on $\Gamma$. Let $\Gamma \rtimes \Delta$ denote the semidirect product constructed via the action of $\Delta$ on $\Gamma$.

If $\Gamma$ is finitely generated, then $\Delta$ is finite. In this case, if $S$ denotes a (finite) set of places including all archimedean places and all places ramified in the finite extension $L / K$, and if $\mathscr{G} / K$ is the discrete, locally algebraic group over $K$ associated to $\Gamma$, then $\mathscr{G} / K$ is the generic fiber of a (unique) locally algebraic group scheme $\mathscr{G} \mid X-S$ which is locally constant for the étale topology over $X-S$.

Definition. A discrete $\operatorname{Gal}(\bar{K} / K)$-group $\Gamma$ (and its associated discrete, locally algebraic group $\mathscr{G} / K)$ is called decent if

(a) the group $\Gamma$ is finitely presented;

(b) the group $\Gamma \rtimes \Delta$ (notation as above) has only a finite number of $\Gamma$ conjugacy classes of finite subgroups.

We will also call a discrete locally algebraic group scheme over $X-S$ decent if its generic fiber is so.

A sub-Gal $(\bar{K} / K)$-group of finite index in a decent $\mathrm{Gal}(\bar{K} / K)$-group is again decent. An inner twist of a decent group is decent. An arithmetic group $\Gamma$ endowed with trivial Galois action is decent.

Lemma (a). If $\mathscr{G}=\mathscr{G} / X-S$ is a decent (discrete) locally constant group scheme, then $H^{1}(X-S, \mathscr{G})$ is finite and (therefore) so is $\mathrm{m}_{S}(\mathscr{G})$.

Proof. Let $\Gamma$ be the associated discrete $\operatorname{Gal}(\bar{K} / K)$-group. Letting $Y$ be the spectrum of the ring of integers of $L$, the splitting field of the natural Galois action on $\Gamma$, and $T$ the inverse image of $S$ in $Y$, we have that $Y-T$ is a "Galois" extension of $X-S$, with group $\Delta=\mathrm{Gal}(L / K)$. We may suppose that $Y-T$ is a finite étale Galois extension of $X-S$.

The set $H^{1}(Y-T, \mathscr{G})$ is finite, for we may identify it with the orbit-space of $\mathrm{Hom}_{\text {cont }}\left(\pi_{1}(Y-T), \Gamma\right)$ under the action of $\Gamma$-conjugation, where $\mathrm{Hom}_{\text {cont }}$ means "continuous homomorphism". Since the image of $\pi_{1}(Y-T)$ in $\Gamma$ under any continuous homomorphism is a finite subgroup of $\Gamma$, by "decency" of $\Gamma$, we have that there are at most a finite number of $\Gamma$-conjugacy classes of finite subgroups of $\Gamma$, and our finiteness assertion follows.

Consequently there is a finite étale Galois extension $Y^{\prime}-T^{\prime} \rightarrow X-S$ admitting $Y-T^{*} \rightarrow X-S$ as an intermediate extension, such that every class in $H^{1}(Y-T, \mathscr{G})$ is trivialized in $Y^{\prime}-T^{\prime}$. Denote by $\Delta^{\prime}$ the Galois group of $Y^{\prime}-T^{\prime}$ over $X-S$ and by $\psi: \Delta^{\prime} \rightarrow \Delta$ the natural surjection. Then $H^{1}(X-S, \mathscr{G})=H^{1}\left(\Delta^{\prime}, \Gamma\right)=\{\Gamma$-conjugacy classes of homomorphisms $\varphi: \Delta^{\prime} \rightarrow \Gamma \rtimes \Delta$ which lift $\left.\psi: \Delta^{\prime} \rightarrow \Delta\right\}$. Since, by the decency hypothesis, the image of $\varphi$ has only a finite number of possibilities up to $\Gamma$-conjugacy, so does $\varphi$.

17. Finiteness theorems. In this section we let $G$ be a smooth locally algebraic group scheme over $X-S$ and $G^{0} \subset G$ the open subgroup scheme which is the "connected component" of $G$ containing the identity section. The quotient $G / G^{0}$ viewed as sheaf for the étale topology over $X-S$ is representable, in general, only as an algebraic space group over $X-S$ (i.e., group object in the 
category of algebraic spaces over $X-S$; cf. [A1, Knu]); even if it is representable as a group scheme, the group scheme does not have to be separated. Denote by $\mathscr{G} / X-S$ the algebraic space group $G / G^{0}$ over the base $X-S$. As usual, we let $\mathscr{G} / K$ denote its generic fiber viewed as (discrete, locally algebraic) a group over $K$.

From now on we shall assume that two further properties hold for $G$ :

(A) The algebraic space group $\mathscr{G} / X-S=G / G^{0}$ is representable as a locally constant group (for the étale topology) over $X-S$ and is decent (cf. $§ 16$ ).

(B) The smooth algebraic group scheme $G^{0} / X-S$ is an extension of an abelian scheme $A / X-S$ by a connected linear affine group scheme.

The second property above is assumed only to tidy up the mental picture we have of our locally algebraic group scheme: it is not really necessary for what we will do and can always be achieved by suitable augmentation of the finite set $S$. Under our assumptions, then, our locally algebraic group scheme $G$ is a successive extension of the following three basic "building blocks":

(a) a discrete, decent, locally constant group scheme $\mathscr{G} / X-S$,

(b) an abelian scheme $A / X-S$,

(c) a connected (smooth) linear affine group scheme $B / X-S$.

We refer below to the subquotient $A$ of $G$ as the abelian scheme part of $G$. Let $A^{0} \subset A$ denote the "connected component" of the Neron model of $A$, i.e., the open subgroup scheme all fibers of which are connected.

Lemma (b). If $A / \mathbb{Q}$ is an abelian variety and $S$ contains all the primes of bad reduction of $A / \mathbb{Q}$ (so that $A$ extends to an abelian scheme over $X-S$ ), then $\mathrm{II}_{S}(A / X-S)$ is isomorphic to the image of $H^{1}\left(X, A^{0}\right)$ in $H^{1}(X-S, A)$. The group $\mathrm{I}_{S}(A / X-S)$ is finite if $\mathrm{\amalg}(A)$ is finite.

Proof. One has a commutative diagram, in which the horizontal rows are exact:

$$
\begin{gathered}
H^{1}\left(X, A^{0}\right) \longrightarrow H^{1}\left(X-S, A^{0}\right) \longrightarrow \\
\downarrow=\prod_{v \in S} H^{1}\left(K_{v}, A^{0}\right) \\
0 \longrightarrow H^{1}(X-S, A) \longrightarrow \mathrm{I}_{S}(A / X-S) \longrightarrow \prod_{v \in S} H^{1}\left(K_{v}, A\right)
\end{gathered}
$$

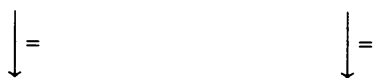

the middle arrow being an equality because all primes of bad reduction for $A$ are assumed to be in $S$. Exactness of the top horizontal row can be seen, for example, from the discussion in the Appendix of [Ma1, p. 263]; exactness of the bottom horizontal row is simply by the definition of $\mathrm{I}_{S}(A / X-S)$. It follows that there is a natural surjection

$$
\alpha: H^{1}\left(X, A^{0}\right) \rightarrow \amalg_{S}(A / X-S) \subset H^{1}(X-S, A) .
$$

Let $\Sigma$ denote the image of $H^{1}\left(X, A^{0}\right)$ in $H^{1}(X, A)$. By the appendix of [Ma1] $\Sigma$ contains $\mathrm{II}(A)$ as a subgroup of finite index (in fact, this index is a power of two). By the above discussion, there is a natural surjection of $\Sigma$ onto $\mathrm{II}_{S}(A / X-S)$.

This proves the lemma.

Lemma (c). If $B / X-S$ is a connected (smooth) linear affine group scheme, then $H^{1}(X-S, B)$ is finite.

Proof. This is [Nis1, Theorem 3.7]; compare also the discussion around [Nis2, Theorem 3.10]. 
To deal with groups which are extensions of these building blocks, consider an exact sequence of locally algebraic smooth group-schemes over a scheme $Y$,

$$
1 \rightarrow U \rightarrow G \stackrel{p}{\rightarrow} W \rightarrow 1 .
$$

If $\gamma \in H^{1}(Y, G)$ is a one-dimensional (noncommutative) cohomology class, let $U_{\gamma}$ be the group scheme $U$ twisted by the class $\gamma$, where $G$ acts on $U$ by conjugation. Let

$$
\pi: H^{1}(Y, G) \rightarrow H^{1}(Y, W)
$$

be the mapping induced from $p$ on one-dimensional (noncommutative) cohomology. If $\omega \in H^{1}(Y, W)$, let $H^{1}(Y, G)_{\omega}$ denote the fiber of $\pi$ above $\omega$. If $\gamma \in H^{1}(Y, G)$ and $\omega=\pi(\gamma)$, by the "long exact sequence" for noncommutative cohomology [Gi, Chapter III, 3.3.5] $]^{34}$, there is a natural surjection of sets

$$
\sigma: H^{1}\left(Y, U_{\gamma}\right) \rightarrow H^{1}(Y, G)_{\omega}
$$

(where the image of the trivial element in $H^{1}\left(Y, U_{\gamma}\right)$ is the element $\gamma$ in $\left.H^{1}(Y, G)_{\omega}\right)$.

Now suppose that $Y=X-S$. We have the diagram

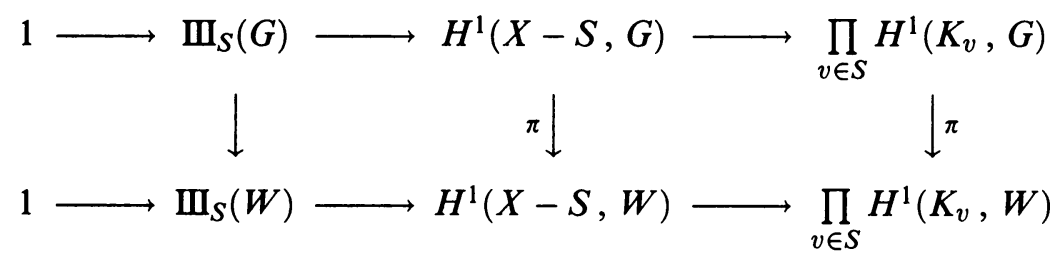

Extension Lemma. Given the exact sequence (1), suppose that

(i) $\mathrm{II}_{S}(W)$ is finite;

(ii) The $\mathrm{W}_{S}\left(U_{\gamma}\right)$ are all finite (for all twisting classes $\gamma \in H^{1}(Y, G)$ ); and either

(iiil) The group scheme $U$ is commutative and the maps $H^{1}\left(K_{v}, U_{\gamma}\right) \rightarrow$ $H^{1}\left(K_{v}, G\right)_{\omega}$ are all proper (for all $v \in S$, and all twisting classes $\gamma \in$ $H^{1}(X-S, G)$, where $\left.\omega=\pi(\gamma)\right)$,

or

(iii2) The $H^{1}\left(X-S, U_{\gamma}\right)$ are all finite (for all twisting classes $\gamma \in$ $\left.H^{1}(X-S, G)\right)$.

Then $\mathrm{I}_{S}(G)$ is finite.

Proof. If $\omega$ is an element in the image of $\mathrm{m}_{S}(G)$ in $\mathrm{m}_{S}(W)$ and if $\gamma \in$ $\mathrm{III}_{S}(G) \subset H^{1}(X-S, G)$ is such that $\omega=\pi(\gamma)$, consider the diagram

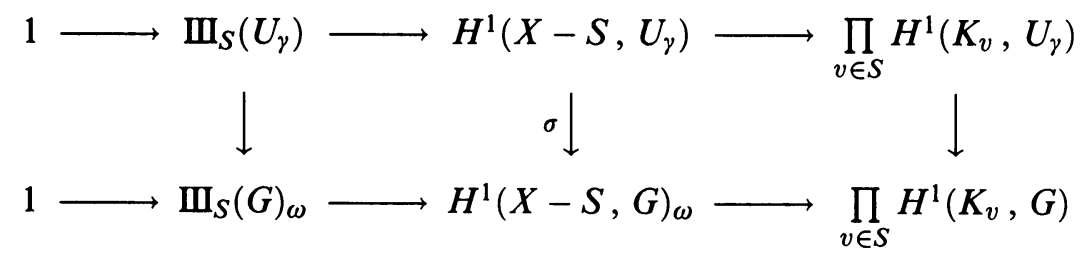

where $\mathrm{II}_{S}(G)_{\omega}$ is defined so as to make the lower sequence exact.

Since, by (i), $\amalg_{S}(W)$ is finite, our lemma will follow if we show $\amalg_{S}(G)_{\omega}$ to be finite for each element $\omega$ in the image of $\mathrm{m}_{S}(G)$ in $\mathrm{m}_{S}(W)$. Fix, then, such a $\omega \in \mathbb{I}_{S}(W)$, and an element $\gamma \in \mathbb{\amalg}_{S}(G)$ such that $\omega=\pi(\gamma)$.

\footnotetext{
${ }^{34}$ For the Galois-cohomological version, compare [Se2, Chapter VII, Appendix, Proposition 2].
} 
From (ii) and (iiil) we see that the "diagonal mapping" of the right-hand rectangle

$$
H^{1}\left(X-S, U_{\gamma}\right) \rightarrow \prod_{v \in S} H^{1}\left(K_{v}, G\right)
$$

has finite kernel. By surjectivity of $\sigma$ we see that this kernel maps onto $\amalg_{S}(G)_{\omega}$ proving the lemma in the case when (iiil) holds.

Now suppose (iii2), giving us finiteness of $H^{1}\left(X-S, U_{\gamma}\right)$. But since $\sigma: H^{1}\left(X-S, U_{\gamma}\right) \rightarrow H^{1}(X-S, G)_{\omega}$ is surjective, finiteness of $\amalg_{S}(G)_{\omega}$ is immediate.

Theorem 4. Let $G$ be a locally algebraic (smooth) group scheme over $X-S$ satisfying our two properties (A) and (B). Suppose, further, that the Tate-Shafarevich Conjecture holds for abelian varieties over $K$. Then $\mathrm{\amalg}_{S}(G)$ is finite.

Remark. If $S \subset S^{\prime}$ is an inclusion of finite sets of places of $K$, and if $G$ is a locally algebraic (smooth) group scheme over $X-S$ satisfying properties (A) and (B), then the restriction of $G$ to $X-S^{\prime}$ also satisfies these properties, so we might amplify the conclusion of our theorem to say that $\mathrm{II}_{S^{\prime}}$ also satisfies these properties, so we might amplify the conclusion of our theorem to say that $\mathrm{II}_{S^{\prime}}(G)$ is finite for $S^{\prime}$ any finite set of places of $K$ containing $S$.

Proof. We do this in two steps.

First we shall assume that our locally algebraic group scheme $G$ fits into an exact sequence of locally algebraic group schemes over $X-S$,

$$
1 \rightarrow U \rightarrow G \stackrel{p}{\rightarrow} W \rightarrow 1
$$

where $W$ is a discrete, decent, locally constant group scheme and $U$ is an abelian scheme. Let us show that, in this situation, $\amalg_{S}(G)$ is finite.

For this, we use the Extension Lemma, noting that property (i) holds by Lemma (a), property (ii) holds by Lemma (b), and our assumption of finiteness of the Tate-Shafarevich groups (of abelian varieties which are "twists" of $A$ ). We now show that property (iiil) holds. We use the long exact sequence of noncommutative cohomology groups and are significantly helped here by the fact that $U$ is an abelian scheme and, in particular, commutative. Properness of the mapping $\varphi: H^{1}\left(K_{v}, U_{\gamma}\right) \rightarrow H^{1}\left(K_{v}, G\right)_{\omega}$ (for all twisting classes $\gamma$ ) is equivalent to showing that the kernels are finite. Let $L_{w} / K_{v}$ be a finite local field extension over which the discrete locally constant group $W$ is constant, i.e., such that the action of $\operatorname{Gal}\left(\bar{L}_{w} / L_{w}\right)$ on $W$ is trivial. We have a commutative square

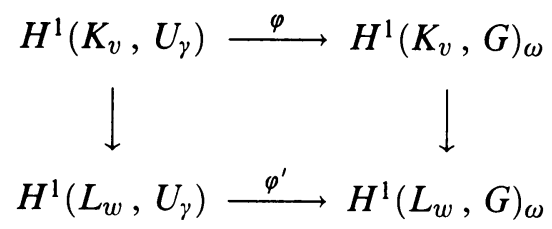

and a coboundary mapping

$$
\delta: H^{0}\left(L_{w}, W\right) \rightarrow H^{1}\left(L_{w}, U_{\gamma}\right)
$$

which is a homomorphism of groups and whose image is the kernel of $\varphi^{\prime}$. To see that $\operatorname{ker} \varphi$ is finite, it suffices to show that the kernel of the diagonal mapping $\psi: H^{1}\left(K_{v}, U_{\gamma}\right) \rightarrow H^{1}\left(L_{w}, G\right)_{\omega}$ in the square above is finite. Since the kernel 
of the left-vertical map $H^{1}\left(K_{v}, U_{\gamma}\right) \rightarrow H^{1}\left(L_{w}, U_{\gamma}\right)$ is finite, finiteness of $\psi$ will follow if we show th $\mathrm{t}$ the image of the homomorphism $\delta$ is finite. But since $W$ is a constant decent group over $L_{w}$, the domain of $\delta, H^{0}\left(L_{w}, W\right)$, is finitely generated. The range of $\delta$ is an abelian torsion group, and therefore the image of $\delta$ is finite. This concludes step 1 .

Now we suppose that our locally algebraic groups scheme $G$ is an extension (1) where $W$ is any locally algebraic group scheme with $\amalg_{S}(W)$ is finite, and where $U$ is a connected linear affine group scheme. In this case, by assumption we have (i) and by Lemma (c) we have (iii2) (which already implies (ii)) so that the Extension Lemma applies again to yield our theorem.

Corollary 1. Let $G$ be a locally algebraic group over $K$ whose group of connected components is decent. Suppose, further, that the Tate-Shafarevich Conjecture holds. Then $\mathrm{\amalg}(G)$ is finite.

Proof. We begin with a lemma on prolongations of locally algebraic groups over $K$ to (smooth) locally algebraic group schemes over $X-S$ (some $S$ ).

Lemma. Let $G$ be a locally algebraic group over $K$ whose group of connected components $\mathscr{G} / K$ is decent. Then $G$ admits an extension to a smooth locally algebraic group scheme, $G / X-S$, satisfying (A) and (B) over $X-S$, for some finite set of places $S$.

Proof. Let $G^{0} / K$ denote the connected component of the identity in $G=G / K$, so that $\mathscr{G} / K=G / G^{0}$ is decent, by our hypothesis. We must, for a suitable finite set of places $S$, construct a "prolongation of $G / K$ ", i.e., a smooth locally algebraic group scheme $G / X-S$ with the property that its generic fiber is $G / K$ and its quotient by $G^{0} / X-S$, the connected component in $G / K$ containing the identity, is locally constant (with generic fiber isomorphic to $\mathscr{G}$ ). We then can achieve (A) and (B) by further augmentation of $S$.

Let $\Gamma=\mathscr{G}(\bar{K})$ be the associated discrete $\operatorname{Gal}(\bar{K} / K)$-group. To construct our prolongation $G / X-S$ we shall not use the full hypothesis of decency of $\mathscr{G}$-only that $\Gamma$ is finitely presented ${ }^{35}$. For simplicity of notation, let us first consider the case where $\Gamma$ is constant rather than only locally constant, when viewed as sheaf for the étale topology over $K$. Let $R \rightarrow F \rightarrow \Gamma \rightarrow 1$ be a finite presentation of $\Gamma$, with $F$ a free group generated by elements $x_{1}, \ldots, x_{n}$ and $R$ a free group generated by elements $y_{1}, \ldots, y_{m}$. By some abuse of notation we may view the natural projection $G \rightarrow \mathscr{G}$ as giving us a homomorphism $G \rightarrow \Gamma$, and let $G^{\sharp}$ denote the fiber-product of $G \rightarrow \Gamma$ and $F \rightarrow \Gamma$, so that we have a commutative diagram

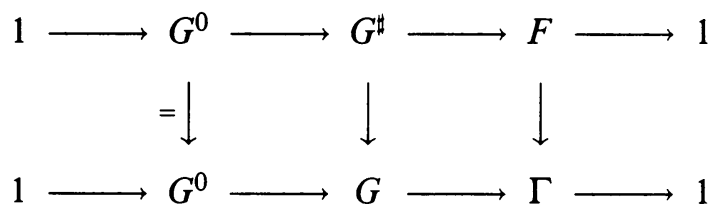

where the rows are exact. For any element $s$ in $F$ (or in $\Gamma$ ) let $G_{s}^{0}$ denote the $G^{0}$-bitorsor in $G^{\sharp}$ (or in $G$ ) which is the full inverse image of $s$. To say that a scheme $Y$ is a bitorsor for a group scheme $H$ means that $Y$ has the structure

\footnotetext{
${ }^{35}$ Ofer Gabber has sketched a construction of such a "prolongation" in the case where $G=$ $\underline{\text { Aut }}(V / K)$ for $V$ a projective smooth variety over $K$ which requires only that $\Gamma$ be finitely generated.
} 
of a left- and a right- $H$-torsor, these actions commuting (cf. [Grot3, Exp. VII] and $[\mathrm{Br}]$ for a treatment of this notion).

First note that $G^{0}$ itself does prolong to a smooth group scheme over $X-S$ (for some finite $S$ ). Fix such a prolongation; call it $G^{0} / X-S$. If $S^{\prime}$ is any finite set containing $S$, let $G^{0} / X-S^{\prime}$ denote the restriction of $G^{0} / X-S$ to $X-S^{\prime}$.

We shall now extend this prolongation of $G^{0}$ to a prolongation of $G^{\sharp}$ to a smooth locally algebraic group scheme over $X-S$ (possibly enlarging $S$ ). For this, consider the $G^{0}$-bitorsors $G_{x_{1}}^{0}, G_{x_{2}}^{0}, \ldots, G_{x_{n}}^{0}$ for the set of generators $x_{1}, \ldots, x_{n}$ of $F$, these all being bitorsors over the base $\operatorname{Spec} K$. Each one of these bitorsors $G_{x_{i}}^{0}$ prolongs to a bitorsor for $G^{0} / X-S_{i}$ for some finite set $S_{i}$ containing $S$. Replacing $S$ by the union of the $S_{i}$ 's, we have bitorsors $G_{x_{i}}^{0} /$ $X-S$ for $G^{0} / X-S$ (for $i=1, \ldots, n$ ). For any word $w$ in $x_{1}, x_{2}, \ldots, x_{n}$ and their inverses in the free group $F$, letting $G_{w}^{0} / X-S$ be the appropriate ("contraction-product" ${ }^{36}$ ) bitorsor for $G^{0} / X-S$ built from the $G_{x_{i}}^{0} / X-S$ one checks that the disjoint union $\amalg G_{w}^{0} / X-S$ taken over all elements $w$ in $F$ (together with multiplication induced by the contraction-product construction) constitutes a prolongation of $G^{\sharp}$ to a locally algebraic group scheme over $X-S$ with the desired properties. Call it $G^{\sharp} / X-S$.

To "descend" this prolongation to a prolongation of $G$ one sees that it suffices to choose trivializations of the $G^{0} / X-S$ bitorsors $G_{y_{j}}^{0} / X-S$ for each of the generators $y_{j}$ of the group of relations $R$, such that these chosen trivializations are "consistent" in the sense that the induced trivialization on $G_{y}^{0} / X-S$ for any $y \in R$ is well defined, i.e., independent of how $y$ is expressed in terms of the generators $y_{j}$. But since $G^{0}$ is separated, "consistency" of these trivializations over $K$ implies "consistency" over $X-S$. We can find such a "consistent" choice of trivializations (for our generating set $y_{j}$ ) over $K$, and then, after a possible further enlargement of $S$ to a finite set of places of $K$, we may extend this choice to $X-S$.

We have then taken care of the case of constant $\mathscr{G}$, i.e., discrete $\mathrm{Gal}(\bar{K} / K)$ groups $\Gamma$ with trivial Galois action. In general though, we have that $\operatorname{Gal}(\bar{K} / K)$ acts on $\Gamma$ continuously, and therefore, recalling the notation of $\S 16$, the action factors through a finite quotient, $\Delta=\operatorname{Gal}(L / K)$. We then begin the procedure as above, i.e., by choosing a prolongation $G^{0} / X-S$ as before, then making the base change to $Y-T$ where $Y$ is the spectrum of the ring of integers in $L$ and $T$ is the inverse image of $S$, and continuing the construction over $Y-T$ appropriately "equivariantly" for the action of $\operatorname{Gal}(L / K)$, and then finally descending back to $X-S$.

Let $G$ denote the extended group scheme given by the preceding lemma. We know by Theorem 4 that $\amalg_{S}(G)$ is finite. Let us define an "intermediate group" (call it $\amalg(S ; G)$ ) which will allow us to compare $\amalg_{S}(G)$ and $\amalg(G)$, namely,

$$
\mathrm{\amalg}(S, G):=\operatorname{ker}\left\{H^{1}(X-S, G) \rightarrow \prod_{v} H^{1}\left(K_{v}, G\right)\right\},
$$

\footnotetext{
${ }^{36}$ This is the noncommutative version of the Baer-sum: one contracts the product of bitorsors by the group action which is the left-action on the right bitorsor and the right-action on the left bitorsor.
} 
where the summation over $v$ is over all places $v$ of $K$. Now, by definition, $\mathrm{W}(S, G)$ is contained in $\mathrm{W}_{S}(G)$ and is consequently also finite. Moreover, if $S \subset T$ is an inclusion of finite sets of places of $v$, the natural mapping $\mathrm{\amalg}(S, G) \rightarrow \amalg(T, G)$ is easily seen to be surjective. Since $\amalg(G)=$ $\underline{\lim } \mathrm{\amalg}(T, G)$, the limit taken over the directed system of all finite sets of places containing $S$, the corollary follows.

Now let $V / K$ be a smooth projective variety. The automorphism group $G=\underline{\operatorname{Aut}}(V / K)$ is a locally algebraic group over $K$. Let $\mathscr{G}=G / G^{0}$ be its (discrete) group of connected components and $\Gamma(V):=\mathscr{G}(\bar{K})$ the group of $\bar{K}$-valued points of $\mathscr{G}$, i.e., the group of connected components of the locally algebraic group $G / \bar{K}=\underline{\operatorname{Aut}}(V / \bar{K})$.

Very little seems to be known, in general, about the groups $\Gamma(V)$. Are they finitely generated for all $V$ ? Finitely presented? decent? Are they all arithmetic groups?

The group $\Gamma(V)$ admits a natural mapping (with finite kernel) to an arithmetic group. ${ }^{37}$ To see this, one uses the natural representation of $G$ on $\mathrm{NS}(V)$, the Néron-Severi group of $V$ which we view as finitely generated abelian locally constant group over $K$, whose full automorphism group is an arithmetic group. One then proves that the subgroup $G_{1}$ of $G$ which is the identity on Néron-Severi is an algebraic group and, in particular, has only a finite number of components.

Let us recall, briefly, how $G_{1}$ is analyzed. First, the action of $G_{1}$ on $V$ gives us an action on $\operatorname{Pic}^{0}(V)$. If $L$ is any line bundle and $g \in G_{1}$, then $\left[g_{*} L \otimes L^{-1}\right]=\left[\left(g^{-1}\right)^{*} L \otimes L^{-1}\right]$ determines a class in $\operatorname{Pic}^{0}(V)$, giving us a 1cocycle $\varphi_{L}$ on the group scheme $G_{1}$ with values in $\operatorname{Pic}^{0}(V)$ defined by $g \mapsto$ $\left[g_{*} L \otimes L^{-1}\right]$. Fixing $L$ to be the very ample line bundle on $V$ coming from a chosen projective embedding, denote by $G_{1, L} \subset G_{1}$ the "kernel" of the 1cocycle $\varphi_{L}$. Define $G_{2, L}$ to be the group (representing the functor) of paired automorphisms of $(V, L)$, i.e., automorphisms of $V$ together with compatible automorphism of $L$. We have a surjection of locally algebraic groups $G_{2, L} \rightarrow$ $G_{1, L}$ (in fact, $G_{2, L}$ is a central extension of $G_{1, L}$ with kernel isomorphic to $\mathbb{G}_{m}$ ). But $G_{2, L}$ is clearly a closed subgroup of the general linear group of automorphisms of $H^{0}(V, L)$. It follows that $G_{1}$ is of finite type, and therefore its image in $\operatorname{Aut}\left(\mathrm{Pic}^{0}(V)\right)$ is finite.

Collecting things, then, we can say that $G=\underline{\operatorname{Aut}}(V / K)$ is a "successive extension" of a subgroup of $\operatorname{Aut}(\mathrm{NS}(V))$ by a subgroup of $\operatorname{Aut}\left(\mathrm{Pic}^{0}(V)\right)$ by a subgroup scheme in $\operatorname{Pic}^{0}(V)$ by a linear group.

Remark. The "abelian variety part" of $G$ is trivial if $\operatorname{Pic}^{0}(V)$ vanishes.

Compare [Ra] where the connected component containing the identity in Aut $(V)$ is discussed.

By [BS, Theorem 2.6], or by the more general "descent theory" (cf. [Grot]) the set $\mathscr{S}(V / K)$ may be identified with $\mathbb{W}(G)$. Hence, we have

Corollary 2. Let $V / K$ be a projective variety such that the locally constant group $\mathscr{G}(V / K)$ of components of $\underline{\operatorname{Aut}}(V / K)$ is decent. Suppose, further, that the Tate-

${ }^{37}$ Under the hypothesis that $\Gamma(V)$ is finitely generated, Ofer Gabber has sketched a proof that $\Gamma(V)$ can be embedded in an arithmetic group. 
Shafarevich Conjecture holds (for all twists of abelian subvarieties of $\operatorname{Pic}^{0}(V / K)$ defined over $K)$. Then $\mathscr{S}(V / K)$ is finite, i.e., the local-to-global principle holds for $V / K$, up to finite obstruction.

Corollary 3. Let $V / K$ be a projective variety such that $\operatorname{Pic}(V / \bar{K})=\mathbb{Z}$. Then $\mathscr{S}(V / K)$ is finite, i.e., the local-to-global principle holds for $V / K$, up to finite obstruction.

Proof. This follows directly from Corollary 2 . For if $\operatorname{Pic}(V / \bar{K})=\mathbb{Z}$, then $\mathscr{G}(V / K)$ is a finite locally constant group scheme and, therefore, is decent. Also, since $\operatorname{Pic}^{0}(V / K)$ vanishes, the hypothesis involving finiteness of the TateShafarevich group of twists of subabelian varieties of $\operatorname{Pic}^{0}(V)$ is certainly satisfied.

Corollary 4. Let $V / K$ be a smooth variety of dimension $\geq 3$ which is a hypersurface (or more generally, a complete intersection) in projective space. Then $\mathscr{S}(V / K)$ is finite, i.e., the local-to-global principle holds for $V / K$, up to finite obstruction.

Proof. For such varieties, $\operatorname{Pic}(V / \bar{K})=\mathbb{Z}$ [Grot2, Corollary 3.7 of Exp. XII] and therefore Corollary 3 applies.

I am thankful to O. Gabber, J. Harris, and J.-P. Serre who told me the proof of the following proposition, which is a result due to Jordan [J] and which implies that, for smooth hypersurfaces $V$ of dimension $\geq 3$ and of degree $\geq 3, \underline{\operatorname{Aut}}(V)$ is, in fact, finite. Therefore, for such varieties the conclusion of Corollary 4 follows directly.

Proposition. Let $n \geq 2$, and let $V$ be a smooth hypersurface in $\mathbf{P}^{n} / \mathbb{C}$ of degree $\geq 3$. Let $\Phi$ denote the subgroup of $\mathrm{PGL}_{n+1}(\mathbb{C})$ which stabilizes $V$. Then $\Phi$ is finite.

Proof. Let $V$ be defined by the homogeneous form $F\left(X_{0}, \ldots, X_{n}\right)$ of degree $d$. The group $\Phi$ is algebraic; it suffices to show that its Lie algebra vanishes. Suppose, then, that $A=\left(a_{i j}\right)(i, j=0, \ldots, n)$ is an $(n+1) \times(n+1)$ matrix in the Lie algebra of $\mathrm{GL}_{n+1}$ which stabilizes $V$, i.e., such that

$$
\sum_{i, j} a_{i j} \cdot X_{j} \cdot \partial F / \partial X_{i}=\lambda \cdot F \quad \text { for } \lambda \in \mathbb{C}^{*} .
$$

Writing $\lambda \cdot F=\lambda \cdot d^{-1} \sum \delta_{i j} \cdot X_{j} \cdot \partial F / \partial X_{i}$ where $\left(\delta_{i j}\right)$ is the identity matrix, we have

$$
\sum_{i, j}\left(a_{i j}-\lambda d^{-1} \delta_{i j}\right) X_{j} \cdot \partial F / \partial X_{i}=0 .
$$

Since $V$ is smooth, the forms $\partial F / \partial X_{i}$ only have the origin in $\mathbb{C}^{n+1}$ as a common intersection, i.e., the sequence

$$
\left(\partial F / \partial X_{0}, \partial F / \partial X_{1}, \ldots, \partial F / \partial X_{n}\right)
$$

is a "regular sequence"; therefore, the ideal of relations of this (regular) sequence is generated by the evident ones

$$
\partial F / \partial X_{i} \cdot \partial F / \partial X_{j}-\partial F / \partial X_{j} \cdot \partial F / \partial X_{i}=0
$$


In other words, the kernel $\mathscr{R}$ of the homomorphism of the $\mathbb{C}\left[X_{0}, \ldots, X_{n}\right]$ algebra $\mathbb{C}\left[X_{0}, \ldots, X_{n}, Y_{0}, \ldots, Y_{n}\right]$ to $\mathbb{C}\left[X_{0}, \ldots, X_{n}\right]$ which sends the variable $Y_{j}$ to $\partial F / \partial X_{j}(j=0, \ldots, n)$ is generated by the elements

$$
\partial F / \partial X_{j} \cdot Y_{i}-\partial F / \partial X_{i} \cdot Y_{j} \quad(i, j=0, \ldots, n) .
$$

Since these elements are homogeneous of degree $d-1 \geq 2$ in the $X_{k}$ 's, it follows that if $\rho_{j}\left(X_{0}, \ldots, X_{n}\right)$ are linear forms in the $X_{k}$ 's for $j=0, \ldots, n$, such that

$$
\sum_{j} \rho_{j}\left(X_{0}, \ldots, X_{n}\right) \cdot \partial F / \partial X_{j}=0
$$

then each of the forms $\rho_{j}\left(X_{0}, \ldots, X_{n}\right)$ vanish identically. Applying this to (2) we have that $a_{i j}=\lambda d^{-1} \delta_{i j}$, i.e., that $A$ gives 0 in the Lie algebra of $\mathrm{PGL}_{n+1}$.

Remarks. Applying this proposition to smooth hypersurfaces of dimension $\geq 3$ and degree $\geq 3$, and using (as in the proof of Corollary 4 ) the fact that, for such varieties, $\operatorname{Pic}(V)=\mathbb{Z}$, we see that $\underline{\operatorname{Aut}}(V)$ is finite. See the discussion of this proposition on [B, pp. 41-42].

Exercise (Serre). What happens when the base field is algebraically closed of characteristic $p$, and $p$ divides the degree $d$ of $V$ ?

What analogous results are there for smooth hypersurfaces in multiprojective spaces?

18. The mapping $\mathrm{I}(A / K) \rightarrow \mathscr{S}(A / K)$. If $A$ is an abelian scheme over a base $S$, to give an automorphism of $A / S$ as abelian scheme is equivalent to giving an automorphism of $A$ viewed merely as scheme over $S$, which fixes the zero-section. Let Aut. $(A)$ denote the group of such automorphisms. Fixing $A / K$, an abelian variety, let Aut. $(A / K)$ denote the locally algebraic group of automorphisms of $A$ preserving zero-section, i.e., of the abelian variety $A$. Let $\mathscr{A}$ denote the set of isomorphism classes of abelian varieties $A^{\prime} / K$ which are isomorphic to $A / K_{v}$ as abelian varieties over $K_{v}$, for all places $v$ of $K$. The set $\mathscr{A}$ is finite [BS, Corollary 7.11]. In fact, $\mathscr{A}$ can be identified with the set $\mathrm{II}$ (Aut. $(A / K))$, and, since Aut $_{\mathrm{f}}(A / K)$ is a locally constant group of "type ALA" in the terminology of [BS], the required finiteness follows.

Theorem 5. The set $\mathscr{S}(A / K)$ has a natural partition into a finite number of (disjoint) subsets indexed by $\mathscr{A}$,

$$
\mathscr{S}(A / K)=\coprod_{\alpha \in \mathscr{A}} \mathscr{S}(A / K)_{\alpha}
$$

and, for any $\alpha \in \mathscr{A}$, if $A^{\prime} / K$ is an abelian variety in the isomorphism class $\alpha$, then there is a natural surjection

$$
\mathrm{\amalg}\left(A^{\prime} / K\right) \rightarrow \mathscr{S}(A / K)_{\alpha}
$$

which identifies $\mathscr{S}(A / K)_{\alpha}$ with the orbit-space of $\amalg\left(A^{\prime} / K\right)$ under the action of the group of automorphisms of $A^{\prime}$ defined over $K$.

Proof. First, let $V$ be a "companion" to $A$, i.e., a projective variety over $K$, isomorphic to $A$ over the completions $K_{v}$ where $v$ ranges over all places of $K$. For a model of the "Albanese variety", $\mathrm{A}_{V}$, of $V$ let us take $\mathrm{A}_{V}=$ 
$\operatorname{Pic}^{0}\left(\operatorname{Pic}^{0}(V)\right)$. Thus $\mathrm{A}_{V}$ is an abelian variety over $K$ isomorphic, as abelian variety, to $A$ over every completion of $K$ and therefore the isomorphism class of the abelian variety $\mathrm{A}_{V}$ over $K$ is an element of $\mathscr{A}$. There is a natural principal homogeneous action of $\mathrm{A}_{V}$ on $V$ (defined over $K$ ). Since $V$ is also a companion to $\mathrm{A}_{V}$ over $K$, it (taken with its structure as principal homogeneous space for $\left.\mathrm{A}_{V}\right)$ determines an element in $\amalg\left(\mathrm{A}_{V}\right)$.

Next, for $\alpha \in \mathscr{A}$, choose an abelian variety $A^{\prime} / K$ in the isomorphism class $\alpha$, and define $\mathscr{S}(A / K)_{\alpha}$ to be the subset of $\mathscr{S}(A / K)$ represented by varieties $V / K$ such that $\mathrm{A}_{V} \cong A^{\prime}$ (the isomorphism being as abelian varieties over $K$ ). This clearly gives a partition of $\mathscr{S}(A / K)$. For each $\alpha \in \mathscr{A}$, we have the required surjection (3), and this mapping visibly factors through the quotient to the orbit-space under the group of $K$-automorphisms of $A^{\prime}$.

To conclude the theorem, we consider two elements in $\mathrm{W}\left(A^{\prime} / K\right)$ which represent the same element in $\mathscr{S}(A / K)_{\alpha}$. Equivalently, on the same projective variety $V$, we are given two principal homogeneous space structures under the abelian variety $A^{\prime}$. We must show that one can bring one of these structures to the other by a $K$-automorphism $\alpha$ of $A^{\prime}$. But this is easy: We get a natural morphism over $K$ from the projective variety $V$ to the (locally constant) group Aut. $\left(A^{\prime} / K\right)$ by the rule which associates to each point $v$ of $V$ the mapping $\varphi_{v}: A^{\prime} \rightarrow A^{\prime}$ given as follows: to any $a \in A^{\prime}$, the translate of $v$ by $a$ via the first principal homogeneous structure is equal to the translate of $v$ by $\varphi_{v}(a)$ via the second principal homogeneous structure. Since $V$ is connected and Aut. $\left(A^{\prime} / K\right)$ is locally constant, the mapping $v \mapsto \varphi_{v}$ is, in fact, constant, and therefore its image is an automorphism $\alpha$ of $A^{\prime}$, defined over $K$.

When is the mapping $\amalg\left(A^{\prime} / K\right) \rightarrow \mathscr{S}(A / K)$ surjective? From the above analysis, this will happen if and only if $\operatorname{\amalg I}\left(\right.$ Aut $\left._{0}(A / K)\right)$ is trivial. Here are two instances:

Corollary 1. $\mathrm{II}\left(A^{\prime} / K\right) \rightarrow \mathscr{S}(A / K)$ is surjective if either:

(a) there is a finite cyclic extension $L / K$ such that every automorphism of $A$ over $\bar{K}$ is already defined over $L$, or

(b) $A=E$ is an elliptic curve.

Proof. Since Aut. $(A / K)$ is discrete, case (a) follows immediately from the corollary to Example (1) in $\S 15$. Case (b) follows from case (a), for if $A$ is an elliptic curve over a number field $K$, the splitting field over $K$ for the action of $\operatorname{Gal}(\bar{K} / K)$ on Aut. $_{.}(A)$ is of degree $\leq 2$.

Corollary 2. For $K$ a fixed number field, the Tate-Shafarevich Conjecture holds for all abelian varieties over $K$ if and only if $\mathscr{S}(A / K)$ is finite for all abelian varieties $A$ over $K$.

Proof. Recall that $\mathscr{A}$ is a finite set. In view of the above theorem, then, it suffices to show that, for any abelian variety, $A / K, \amalg(A / K)$ is finite if and only if its image in $\mathscr{S}(A / K)$ is finite, or, equivalently, if and only if there are only a finite number of orbits of $\amalg(A / K)$ under the action of the $K$ automorphism group of $A$. But, as Tate remarked to me, this is easy to see: there are only a finite number of elements in $\mathrm{II}(A / K)$ of any fixed order, and automorphisms of groups preserve the order of elements. 
19. The local-to-global principle for quadrics. I am thankful to Colliot-Thélène for showing me this proof of the local-to-global principle for any smooth quadric $V$ over a number field $K$.

Let $W / K$ be a smooth projective variety which is potentially quadric in the sense that its base change to $\bar{K}$ is isomorphic to a quadratic hypersurface in projective space. Putting $\bar{W}=W \times_{\operatorname{Spec} K} \operatorname{Spec} \bar{K}$ we have that $\operatorname{Pic}(\bar{W})$ is isomorphic either to $\mathbb{Z}$ or $\mathbb{Z} \oplus \mathbb{Z}$ (the latter only if $\bar{W}$ is of dimension 2 ). Call an element $\xi$ of $\operatorname{Pic}(\bar{W})$ of quadric type if a line bundle $\overline{\mathscr{L}}$ over $\bar{W}$ representing $\xi$ has the property that $\overline{\mathscr{L}}$ is very ample and the embedding of $\bar{W}$ in projective space associated to the complete linear system of $\overline{\mathscr{L}}$ identifies $\bar{W}$ with a quadric hypersurface over $\bar{K}$. There is only one element $\xi$ "of quadric type" in $\operatorname{Pic}(\bar{W})$, and that element $\xi$ is fixed under the action of $\operatorname{Gal}(\bar{K} / K)$. We have the exact sequence

$$
0 \rightarrow \operatorname{Pic}(W) \rightarrow \operatorname{Pic}(\bar{W})^{\mathrm{Gal}(\bar{K} / K)} \underset{\delta_{K}}{\rightarrow} \operatorname{Br}(K)
$$

where $\operatorname{Br}(K)$ is the Brauer group of $K$ and $\delta_{K}$ is the natural "coboundary" mapping whose definition is as follows. For a line bundle $\bar{l}$ on $\bar{W}$ whose isomorphism class is fixed by $\mathrm{Gal}(\bar{K} / K)$ and for each $\sigma \in \mathrm{Gal}(\bar{K} / K)$ choose an isomorphism $l_{\sigma}: \bar{l} \cong \bar{l}^{\sigma}$. Denote by $c$ the 2-cocycle on $\operatorname{Gal}(\bar{K} / K)$ with coefficients in $\bar{K}^{*}$ defined by the formula $c(\sigma, \tau)=\left(l_{\sigma \tau}\right)^{-1} \cdot\left(l_{\sigma}\right)^{\tau} \cdot l_{\tau}$. Then if $[\bar{l}]$ denotes the isomorphism class of $\bar{l}$ in $\operatorname{Pic}(\bar{W})^{\mathrm{Gal}(\bar{K} / K)}, \delta_{K}([\bar{l}])$ is defined to be the cohomology class of $c$ in the Brauer group $\operatorname{Br}(K)=H^{2}\left(\operatorname{Gal}(\bar{K} / K) ; \bar{K}^{*}\right)$; this cohomology class is seen to be dependent only upon the isomorphism class of $[l]$ and independent of the choice of the $l_{\sigma}$ 's.

The element $\delta_{K}(\xi) \in \operatorname{Br}(K)$ may be thought of as the obstruction to $W / K$ being isomorphic to a quadric hypersurface over $K$, for its vanishing is a necessary and sufficient condition for the line bundle $\overline{\mathscr{L}}$ corresponding to $\xi$ on $\bar{W}$ to come from a line bundle $\mathscr{L}$ on $W / K$ whose linear system would identify $W$ with a quadric hypersurface in projective space over $K$.

If $V$ is a smooth quadric over $K$ and $W$ a companion to $V$ over $K$, then $V$ is isomorphic to $W$ over $\bar{K}$, so $W$ is potentially quadric. Since $V / K_{v}$ is isomorphic $W / K_{v}$ for all places $v$ of $K, W$ is, in fact, quadric over $K_{v}$ for all $v$; therefore, it follows from the version of (4) over $K_{v}$ that $\delta_{K_{v}}(\xi)=0$ in $\operatorname{Br}\left(K_{v}\right)$ for all $v$. Since the global Brauer group injects into the direct sum of the local Brauer groups, $\delta_{K}(\xi)=0$; therefore, the companion $W$ is a quadric variety over $K{ }^{38}$ Now let $q_{V}$ and $q_{W}$ denote the two quadratic forms over $K$ corresponding to $V$ and $W$. Since $V$ and $W$ are companions, it follows (from the argument alluded to in the footnote to $\S 2$ of Part I) that the corresponding forms $q_{V, v}$ and $q_{W, v}$ over the completions $K_{v}$ for each place $v$ of $K$ are similar. Using [O] we then see that $q_{V}$ and $q_{W}$ are similar over $K$.

\section{ACKNOWLEDGMENT}

I am thankful to M. Artin, P. Diaconis, J.-L. Colliot-Thélène, O. Gabber, J. Harris, N. Katz, Y. Nisnevich, K. Rubin, and J.-P. Serre for their generous

\footnotetext{
${ }^{38} \mathrm{~A}$ similar argument shows that a smooth projective variety $W / K$ of dimension $\geq 3$ which is a companion to a hypersurface of degree $d$ defined over $K$ is itself isomorphic over $K$ to a hypersurface of degree $d$.
} 
help and to the audiences at the MSRI ${ }^{39}$, the University of Toronto, and Penn State $^{40}$, for their comments, suggestions, and patience during my talks on material corresponding to Part I of this article.

\section{BIBLIOGRAPHY}

[A1] M. Artin, Algebraic spaces, Yale Math. Monographs, vol. 3, Yale Univ. Press, New Haven, CT, 1976.

[A2] M. Artin, Néron models, Arithmetic Geometry (G. Cornell and J. Silverman, eds.), Springer-Verlag, New York, 1986, pp. 213-230.

[BD] M. Bertolini and H. Darmon, Kolyvagin's descent and Mordell-Weil groups over ring class fields, J. Reine Angew. Math. 412 (1990), 63-74.

[B] A. Borel, Introduction aux groupes arithmétiques, Hermann, Paris, 1969.

[BS] A. Borel and J.-P. Serre, Théorèmes de finitude en cohomologie galoisienne, Comment. Math. Helv. 39 (1964), 111-164, Oeuvres. Collected papers, vol. II 1959-1968, SpringerVerlag, New York, 1983, pp. 362-415.

[BLR] S. Bosch, W. Lütkebohmert, and M. Raynaud, Néron models, Ergeb. Math. Grenzgeb., bd. 21, Springer-Verlag, New York, 1990.

[Br] L. Breen, Bitorseurs et cohomologie non abélienne, The Grothendieck Festschrift, vol. II (A collection of articles written in honor of the 60th birthday of Alexander Grothendieck), Birkhäuser, Basel, 1991, pp. 399-476.

[Ca1] J. W. S. Cassels, Rational quadratic forms, Academic Press, New York, 1978.

[Ca2] Lectures on elliptic curves, London Math. Soc. Stud. Texts, vol. 24, Cambridge Univ. Press, London and New York, 1991.

[Ch] V. Chernousov, On the Hasse principle for groups of type $E_{8}$, Soviet Math. Dokl. 39 (1989), 592-596.

[Coa] J. Coates, Elliptic curves with complex multiplication and Iwasawa theory, Bull. London Math. Soc. 23 (1991), 321-350.

[Coh] P. J. Cohen, Decision procedures for real and p-adic fields, Comm. Pure Appl. Math. 22 (1969), 131-151.

[C-T] J.-L. Colliot-Thélène, Les grands thèmes de François Châtelet, Enseign. Math. 34 (1988), 387-405.

[C-T-S-D] J.-L. Colliot-Thélène and P. Swinnerton-Dyer, Hasse principle and weak approximation for pencils of Severi-Brauer and similar varieties, preprint, 1992.

[D1] H. Darmon, Euler systems and refined conjectures of Birch Swinnerton-Dyer type, Proc. Conf. p-adic Birch Swinnerton-Dyer Conjectures (Boston Univ., August 1991) (to appear).

[D2] A A refined conjecture of Mazur-Tate type, Invent. Math. 110 (1992), 123-146.

[Du] W. Duke, Hyperbolic distribution problems and half-integral weight Maass forms, Invent. Math. 92 (1988), 73-90.

[D-S-P] W. Duke and R. Schulze-Pillot, Representation of integers by positive ternary quadratic forms and equidistribution of lattice points on ellipsoids, Invent. Math. 99 (1990), 49-57.

[F] M. Flach, A finiteness theorem for the symmetric square of an elliptic curve, Invent. Math. 109 (1992), 307-327.

[Gi] J. Giraud, Cohomologie non abélienne, Grundlehren Math. Wissen., bd. 179, SpringerVerlag, New York, 1971.

[GF] E. Golubeva and O. Fomenko, Application of spherical functions to a certain problem in the theory of quadratic forms, J. Soviet Math. 38 (1987), 2054-2060.

\footnotetext{
${ }^{39}$ At the conference in June 1992 commemorating the 10th anniversary of the MSRI and in honor of Irving Kaplansky on the occasion of his retirement as director.

${ }^{40}$ The Weisfeiler Lecture, November 1992.
} 
[Gros] B. Gross, Kolyvagin's work on modular elliptic curves, L-functions and Arithmetic (J. Coates and M. J. Taylor, eds.), Proc. Durham 1989, London Math. Soc. Lecture Note Ser., vol. 153, Cambridge Univ. Press, London and New York, 1991.

[Grot1] A. Grothendieck, Techniques de descente et théorèmes d'existence en géométrie algébrique, Sém. Bourbaki, vol. 12 (59/60), Benjamin Inc., New York and Amsterdam, 1966.

[Grot2] A. Grothendieck (ed.), Cohomologie locale des faisceaux cohérents et Théorèmes de Lefschetz locaux et globaux (SGA 2), Advanced Studies in Pure Mathematics, vol. 2, North-Holland, Amsterdam, 1968.

[Grot3] A. Grothendieck (ed.), Groupes de monodromie en géométrie algébrique (SGA 7 I), Séminaire de Géométrie Algébrique du Bois-Marie 1967-1969, Lecture Notes in Math., vol. 288, Springer-Verlag, New York, 1972.

[Hard] G. Harder, Über die Galoiskohomologie halbeinfacher Matrizengruppen, Math. Z. 90 (1965), 404-428; 92 (1966), 396-415.

[Harr] J. Harris, Algebraic geometry (a first course), Graduate Texts in Math., vol. 133, Springer-Verlag, New York, 1992.

[Hart] R. Hartshorne, Algebraic geometry, Springer-Verlag, New York, 1977.

[Has] H. Hasse, Über die Äquivalenz quadratischer Formen im Körper der rationalen Zahlen, J. Reine Angew. Math. 152 (1923), 205-224.

[I] H. Iwaniec, Fourier coefficients of modular forms of half integral weight, Invent. Math. 87 (1987), 385-401.

[J] C. Jordan, Mémoire sur Pequivalence des formes, J. École Polytech XLVIII (1880), 112-150.

[KO] S. Kobayashi and T. Ochiai, Mappings into compact complex manifolds with negative first Chern class, J. Math. Soc. Japan 23 (1971), 136-148.

[K1] V. A. Kolyvagin, Finiteness of $E(\mathbb{Q})$ and $\mathrm{III}(\mathbb{Q})$ for a subclass of Weil curves, Izv. Akad. Nauk SSSR Ser. Mat. 52 (1988), 522-540; English transl., Math USSR-Izv. 32 (1989), 523-542.

[K2] _ On the Mordell-Weil group and the Shafarevich-Tate group of Weil elliptic curves, Izv. Akad. Nauk SSSR Ser. Mat. 52 (1988), 1154-1180.

[K3] Euler systems, The Grothendieck Festschrift, vol. II (A collection of articles written in honor of the 60th birthday of Alexander Grothendieck), Birkhäuser, Basel, 1991 , pp. $435-483$.

[KL] V. A. Kolyvagin and D. Logachev, Finiteness of the Shafarevich-Tate group and group of rational points for some modular abelian varieties, Algebra i Analiz 1 (1989), 171-196; English transl., Leningrad Math. J. 1 (1990), 1229-1253.

[Kne] M. Kneser, Lectures on Galois cohomology of classical groups, Tata Inst. Fund. Res. Lectures on Math. and Phys., Tata Inst. Fund. Res., Bombay, 1969.

[Knu] D. Knutsen, Algebraic spaces, Lecture Notes in Math., vol. 203, Springer-Verlag, New York, 1971.

[Ma1] B. Mazur, Rational points of abelian varieties with values in towers of number fields, Invent. Math. 18 (1972), 183-266.

[Ma2] _ Number theory as gadfly, Amer. Math. Monthly 98 (1991), 593-610.

[Mat] Y. Matijasevic, Enumerable sets are diophantine, Dokl. Akad. Nauk SSSR 191 (1970); English transl., Soviet Math. Dokl. 11 (1970), 354-358.

[Mi1] J. Milne, The conjectures of Birch and Swinnerton-Dyer for constant abelian varieties over function fields, Thesis, Harvard Univ., 1967.

[Mi2] The Tate-Shafarevich group of a constant abelian variety, Invent. Math. 5 (1968), 63-84. , On a conjecture of Artin and Tate, Ann. of Math. (2) 102 (1975), 517-533.

[Mo] L. J. Mordell, Diophantine equations, Academic Press, New York, 1969. 
[Ne1] J. Nekováŕ, Kolyvagin's method for Chow groups of Kuga-Sato varieties, Invent Math. 107 (1992), 99-125.

[Ne2] _ p-Adic methods in arithmetic, Center for Pure and Appl. Math., Univ. of California, Berkeley, preprint, 1992.

[Né] A. Néron, Modèles minimaux des variétés abéliennes sur les corps locaux et globaux, Inst. Hautes Études Sci. Publ. Math. 21 (1964).

[Nik1] V. Nikulin, On factor groups of the automorphism groups of hyperbolic forms modulo subgroups generated by 2-reflections, Soviet Math. Dokl. 20 (1979), 1156-1158.

[Nik2] , Quotient-groups of the automorphisms of hyperbolic forms by subgroups generated by 2-reflections, algebro-geometric applications, Current Problems in Math., vol. 18, Akad. Nauk SSSR, Usesoyuz. Inst. Nauchn. Tekhn. Informatsii, Moscow, 1981, pp. 3-114; English transl., J. Soviet Math. 22 (1983), no. 4.

[Nis1] Y. Nisnevich, Etale cohomology and arithmetic of semi-simple groups, Ph.D. thesis, Harvard Univ., 1982.

[Nis2] - On certain arithmetic and cohomological invariants of semi-simple groups, Johns Hopkins Univ., preprint, 1989.

[O] T. Ono, Arithmetic of orthogonal groups, J. Math. Soc. Japan 7 (1955), 79-91.

[P] B. Perrin-Riou, Travaux de Kolyvagin et Rubin, Sém. Bourbaki, exp. 717 (1989/90); Astérique 189-190 (1990), 69-106.

[P-S-S] I. Piatetski-Shapiro and I. Shafarevich, $A$ Torelli theorem for algebraic surfaces of type K3 , Math. USSR-Izv. 5 (1971), 547-588.

[Pl] V.P. Platonov, The arithmetic theory of algebraic groups, Uspekhi Mat. Nauk 37 (1982), 3-54; English transl., Russian Math Surveys 37 (1982), 1-62.

[Ra] C. P. Ramanujam, A note on automorphism groups of algebraic varieties, Math. Ann. 19 (1964), 25-33.

[R1] K. Rubin, Tate-Shafarevich groups and L-functions of elliptic curves with complex multiplication, Invent. Math. 89 (1987), 527-560.

[R2] On the main conjecture of Iwasawa theory for imaginary quadratic fields, Invent. Math. 93 (1988), 701-713.

[R3] Kolyvagin's system of Gauss sums, Arithmetic Algebraic Geometry (van der Geer, Oort, and Steenbrink, eds.), Progr. Math., vol. 89, Birkhäuser, Boston, MA, 1991, pp. 309-324.

[R4] The main conjecture, Appendix to S. Lang's Cyclotomic Fields I and II, combined second ed., Graduate Texts in Math., vol. 121, Springer-Verlag, New York, 1990, pp. 397-429.

[R5] The "main conjectures" of I wasawa theory for imaginary quadratic fields, Invent. Math. 103 (1991), 25-68.

[R6] The one-variable main conjecture for elliptic curves with complex multiplication, $L$-functions and Arithmetic (J. Coates and M. J. Taylor, eds.), Proc. Durham 1989, London Math. Soc. Lecture Note Ser., vol. 153, Cambridge Univ. Press, London and New York, 1991.

[R7] , Stark units and Kolyvagin's “Euler systems”, J. Reine Angew. Math. 425 (1992), 141-154.

[R8] The work of Kolyvagin on the arithmetic of elliptic curves, Arithmetic of Complex Manifolds, Lecture Notes in Math., vol. 1399, Springer-Verlag, New York, 1989, pp. 128-136.

[Se1] J.-P. Serre, Géométrie algébrique et géométrie analytique, Ann. Inst. Fourier (Grenoble) 6 (1956), 1-42.

[Se2] Cohomologie galoisienne (3rd. ed.), Lecture Notes in Math., vol. 5, SpringerVerlag, New York, 1965.

[Se3] _ Local fields, Springer-Verlag, New York, 1979. 
[Si] C. Siegel, Equivalence of quadratic forms, Amer. J. Math. 63 (1941), 658-680; Carl Ludwig Siegel Gesammelte Abhandlungen, bd. II, Springer-Verlag, New York, 1966, pp. 217-239.

[St] N. Stephens, The diophantine equation $X^{3}+Y^{3}=D Z^{3}$ and the conjectures of Birch and Swinnerton-Dyer, J. Reine Angew. Math. 231 (1968), 121-162.

[Ta] J. Tate, The arithmetic of elliptic curves, Invent. Math. 23 (1974), 179-206.

[Th] F. Thaine, On the ideal class groups of real abelian number fields, Ann. of Math. (2) 128 (1988), 1-18.

[W] A. Weil, Sur lanalogie entre les corps de nombres algébriques et les corps de fonctions algébriques (1939), Oeuvres Sci. I, Springer-Verlag, New York, 1980, pp. 236-240.

Department of Mathematics, Harvard University, 1 Oxford Street, Cambridge, MasSACHUSETTS 02140

E-mail address: mazur@math.harvard.edu 